\title{
Enabling Scalable and Accurate Clustering of Distributed Ligand Geometries on Supercomputers
}

\author{
Boyu Zhang ${ }^{\mathrm{a}, \mathrm{b}}$, Trilce Estradac ${ }^{\mathrm{c}}$, Pietro Cicotti $^{\mathrm{d}}$, Pavan Balaji ${ }^{\mathrm{e}}$, Michela \\ Taufer $^{\mathrm{f}, *}$ \\ ${ }^{a}$ University of Delaware \\ ${ }^{b}$ Purdue University \\ ${ }^{c}$ University of New Mexico \\ ${ }^{d}$ San Diego Supercomputer Center \\ ${ }^{e}$ Argonne National Laboratory \\ ${ }^{f}$ University of Delaware
}

\begin{abstract}
We present an efficient and accurate clustering method for the analysis of protein-ligand docking datasets on large distributed-memory systems. For each ligand conformation in the dataset, our clustering algorithm first extracts relevant geometrical properties and transforms the properties into a single metadata point in the N-dimensional (N-D) space. Then, it performs an N-D clustering on the metadata to search for predominant clusters. Our method avoids the need to move ligand conformations among nodes, because it extracts relevant data properties locally and concurrently. By doing so, we transform the analysis problem (e.g., clustering or classification) into a search for property aggregates. Our analysis shows that when using small computer systems of up to 64 nodes, the performance is not sensitive to data content and distribution. When using larger computer systems of up to 256 nodes the scalability of simulations with strong convergence toward specific geometries is less sensitive to overheads due to the shuffling of metadata information. We also demonstrate that our method of metadata extraction captures the geometrical properties of ligand conformations more effectively and clusters and predicts near-native ligand conformations more accurately than do traditional methods, including the hierarchical cluster-
\end{abstract}

\footnotetext{
* Corresponding author

Email address: taufer@acm.org (Michela Taufer)
}

Preprint submitted to Journal of Parallel Computing

November 26, 2016

(C) 2017. This manuscript version is made available under the Elsevier user license http://www.elsevier.com/open-access/userlicense/1.0/ 
ing and energy-based scoring methods.

Keywords: Ligand conformations, Protein-ligand docking, Drug design, Octree-based clustering, N-dimensional clustering, In situ analytics.

\section{Introduction}

In studies of disease processes, a common problem involves the search for small molecules (ligands) that can interact with a larger molecule, such as a protein, that is involved in a disease state. Specifically, when ligands dock

5 well in a protein, they can potentially be used as a drug to stop or prevent diseases associated with the protein malfunction. The study of the docking process is computationally performed with docking simulations, which consist of sequences of independent docking trials. Each docking trial generates a series of random initial ligand conformations and orientations. These conformations can be docked into the protein-binding site (or pocket) through molecular dynamics simulated annealing [1]. Since the process is highly parallelizable, it is efficiently performed on distributed-memory systems (e.g., supercomputers and volunteer computing platforms), resulting in a distributed collection of hundreds of thousands of ligand conformations across the nodes of the system. The docking simulations often produce large datasets of ligand conformations that naturally converge toward native conformations (i.e., conformations experimentally observed).

When analyzing large datasets searching for the native conformations, a common practice is to reduce the number of candidates up to 100 conformations based on energy values and then leave the scientists with the tedious task of subjectively selecting a possible near-native ligand. Scientists normally perform this task manually by using visual tools such as VMD 2] or Chimera [3]. Then the scientists perform wet-lab high-throughput screening on the selected ligands. Not only does the manual selection process still depend on inaccurate energy scoring but it also can be highly error-prone. To the best of our knowledge, most advanced methods of handling this task are not fully automated, and the 
need remains not only for automation of this process but also for faster selecting methodology.

In previous work 4, we addressed the problem of accurately and automatso ically selecting near-native conformations from the dataset by using a probabilistic hierarchical clustering based on ligand geometries. Unfortunately the method in [4] requires direct comparison of the conformations' geometries iteratively. When very large numbers of conformations are distributed across a large number of nodes of the distributed-memory system, the movement and comparison of data can have a significant negative impact on the performance and scalability of the analysis.

In this paper we move away from the iterative process used in [4] and propose a more efficient and accurate method to cluster conformation geometries across the nodes of a distributed-memory system that does not require direct movement and comparison of ligand conformations. The method used in this paper was initially presented in [5, 6, 7,. This paper gathers the contributions of the publications in a comprehensive manuscript. More important, this paper substantially extends performance results presented at the IEEE International Scalable Computing Challenge (SCALE 2015) 7] and adds accuracy results not 45 presented before.

Our method first maps the relevant geometrical properties of each conformation into a concise metadata point in the N-dimensional (or N-D) space (i.e., a single point in a lower-dimensional space). Our lower-dimensional representation can be viewed as metadata since it encodes the geometries of the original ligand conformations. The geometrical properties quantify the direction and position of the ligand confirmations in the fixed, known docking pocket of the protein. Then, our method performs an N-D clustering on all metadata points to search for dense clusters. Our hypothesis is that the method accurately maps ligand conformations with similar geometries to metadata in the N-D space in 55 close proximity. Thus subspaces of the metadata space with higher point concentrations (or densities) can be associated with most frequently found ligand conformations in a docking simulation that naturally converges toward the na- 
tive conformation. For mapping conformation geometries into metadata points, we consider three variations of an algorithm that uses projections and linear interpolations into 3-D, 3-Dlog, and 6-D mappings. To search for the densest and deeper subspace in the N-D space of metadata points, we consider two variations of an N-D tree search. In the first variation, called GlobalToLocal (GL), the extracted properties are moved across nodes to build a global view of the dataset; these properties are iteratively and locally analyzed while searching ToGlobal (LG), partial properties are analyzed locally on each node to generate a property aggregate, which is a scalar number that summarizes local properties of the data. In this variation, instead of exchanging extracted properties, LG exchanges only a few scalar property aggregates across the nodes. Both 70 approaches are based on the general theme of moving computation to the data. Figure 1 shows an overview of our approach for one of the three variations for mapping conformation geometries in which each conformation geometry is mapped into a single 3 -D point and the N-D search is performed on an octree. By mapping atomic coordinates of a ligand into a single-point metadata, our clustering method enables in situ analysis of ligand conformations in proteinligand docking simulations due to the following three aspects: our clustering method executes sufficiently fast comparing to the docking simulation, it avoids moving actual ligand coordinates data, and it limits the memory use of ligand conformations by keeping only $3-\mathrm{D}$ or 6 -D metadata points in memory.

Our performance study assesses our method at both small and large scales. At the small scale (up to 64 compute nodes), we measure the performance of the GL and LG variations and assess their sensitivity to both logical and physical data distributions. Because we discovered that the GL variation exhibits poor scalability already on a cluster of 64 compute nodes, we focus our present study 85 at the larger scale (up to 256 nodes) on the LocalToGlobal variation only and identify the crucial features in a logical distribution that more significantly impact the execution times when performing the search on large datasets of ligand conformations. 


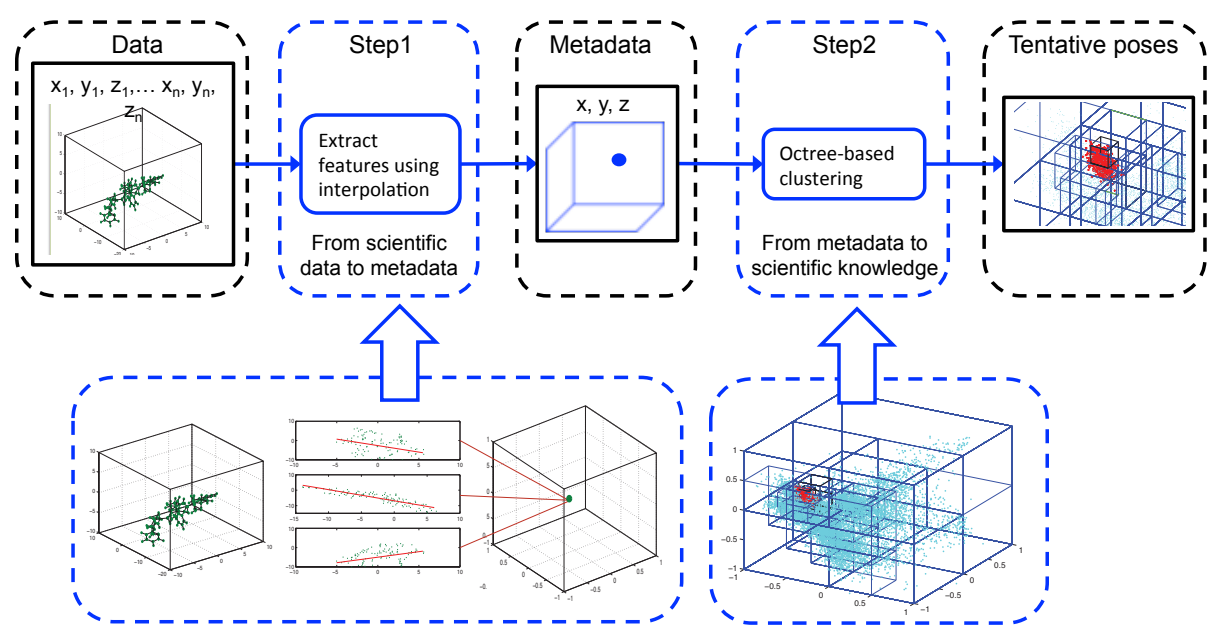

Figure 1: Overview of ligand clustering in which ligand conformations are mapped into metadata of 3-D points and metadata points are searched for knowledge acquisition.

Our accuracy study aims to quantify the capability of our proposed mapping variations (i.e., the 3-D, 3-Dlog, and 6-D mappings) to capture and preserve ligand conformation geometries. In other words, we assess whether our transformation of conformations into simple metadata points preserves the knowledge of the conformations geometries and to determine whether we can still acquire this knowledge from the most frequently sampled geometries despite their generation across multiple nodes of a distributed-memory system. To this end we analyze the ligand conformations generated by real large-scale protein-ligand docking simulations within the Docking@Home project. This project considers a diverse set of proteins and ligands from the LPDB database and performs extensive docking attempts [8].

The paper is organized as follows. Section 2 gives the background knowledge on protein-ligand docking simulations. Section 3 reviews related work on the clustering techniques of ligand conformations and the distributed clustering using MapReduce. Section 4 discusses our method and its variations of mapping and N-D search. Sections 5 and 6 present the performance and accuracy evaluations, respectively. Section 7 summarizes our conclusions and briefly describes 
future work.

\section{Background}

This section provides a comprehensive view of the science that is enabled by our scalable and accurate clustering method as well as a short overview of the is not always the case [5]. Since selecting the near-native ligand geometry based on energy alone may result in incorrect conclusions, an alternative approach 
selects the near-native geometry from clustering. In previous work we showed

that compact clusters of docked conformations grouped by their geometries are more likely to be near-native than are the individual conformations with lowest energy [5, 11. Large numbers of ligand conformations were sampled through the Docking@Home $(\mathrm{D} @ \mathrm{H})$ project in the past five years and are used here as the dataset of interest. Hundreds of millions of docked ligand conformations must be compared with each other in terms of their geometries. However, this approach can result in extensive computing and storage needs. Ideally the clustering methods have to be scalable, efficient, and accurate, allowing scientists to compare and select across a large set of docking results.

\subsection{Generating large datasets with Docking@Home}

Although the system software for generating the large datasets of conformations used for our analysis is not our key contribution here, we briefly describe how we collect the docking data for this study using the Docking@Home project [12]. Docking@Home is one of the many computational molecular docking approaches for sampling large conformational spaces of ligands that have been used for virtual screening [13, 14, 15]. Typically a given docking method is evaluated with a selected number of experimentally determined protein-ligand complexes. In general, docking methods differ from each other in the algorithm used in the conformational search [16, 17, the scoring function used to predict ligand geometries, and the scoring function used to rank compounds (or predict DGbinding) [18.

Docking@Home (D@H) uses the Volunteer Computing (VC) programming model to build the framework of distributed computing resources for the docking simulations; ordinary people volunteer processing and storage resources across the Internet to contribute to the scientific simulations. $\mathrm{D} @ \mathrm{H}$ builds on top of Berkeley Open Infrastructure for Network Computing (BOINC) 19, wellknown VC middleware. D@H computationally searches for potential drug-like molecules against diseases such as breast cancer and HIV. D@H generates a large space of possible docking conformations. In order to extensively search 
this space, millions of independent docking attempts (jobs) are processed by mation. Random conformations are generated starting from the ligand crystal structure with random initial velocities on each ligand atom. Then the initial conformation is randomly rotated to produce a set of orientations that are 
placed into the active site of the protein or docking pocket (docking attempts).

Once the ligand is docked into the protein site, a molecular dynamics simulation is performed consisting of a gradual heating phase of 4,000 1 femtosecond (fs) steps from $300 \mathrm{~K}$ to $700 \mathrm{~K}$, followed by a cooling phase of 10,000 1 fs steps back to $300 \mathrm{~K}$. In order to facilitate the penetration of ligands into protein sites and allow larger conformational changes, van der Waals (vdW) and electrostatic potentials with soft-core repulsions are utilized. A soft-core repulsion reduces the potential barrier at vanishing interatomic distances to a finite limit, allowing ligands to pass between conformational minima with a relatively small potential barrier that normally is large and impossible to overcome with an unmodified standard potential. The detailed description of the docking method and its comparison with other docking codes are not in the current scope of this work; details can be found in [22, 1]. Once the results (ligand conformations) are collected, they need to be scored. Initially D@H used an energy-based scoring method; our previous work pointed out how this scoring approach can result in incorrect conclusions because energy values are approximated by the simplified methods used in the computational algorithms. The alternative approach we pursue scores ligands based on the geometry of their resulting conformations.

\subsection{Semi- and fully decentralized memory systems}

The data considered here comprises a large number of individual data records and is distributed across the nodes of a large distributed-memory system. More specifically, we consider semi- and fully decentralized distributed-memory systems. In a semi-decentralized system, processes report to more than one node, usually to the closest one in the cluster's network topology, and take advantage of locality by reducing expensive data transfer and potential storage pressure.

In contrast, in a fully decentralized system, each node stores its own data, which reduces the need for data transfers and increases the amount of locally stored data. Logically, the entire dataset can converge toward one or multiple scientific properties, or it may not convey any scientific information. Physically, data with similar scientific properties may agglomerate in topologically close 
nodes or may be dispersed across nodes. Logical and physical tendencies are not known a priori when data is generated in semi- or fully decentralized systems. In general, scientists must move data across nodes in order to analyze and understand it. This process can be extremely costly in terms of execution time. For completeness, we define three scenarios that resemble the challenging conditions faced by scientists when dealing with distributed systems with large amounts of data. The scenarios consist of data distributed as follows:

- a semi-decentralized manner in which data with similar properties are generated by and stored in specific nodes;

- a fully decentralized, synchronous manner in which data is gathered at regular intervals producing a uniform distribution of data properties across the nodes in a round-robin fashion; and

- a fully decentralized, asynchronous manner in which every node acts by itself and properties are stored randomly across nodes.

\subsection{MapReduce-MPI and other MapReduce libraries}

MapReduce-MPI is a runtime library supporting the MapReduce programming model [23]. It is written in $\mathrm{C}++$ and MPI. It runs a MapReduce program using a number of MPI processes, the number of which is defined by the programmer. Each process runs both the map and the reduce functions. It first runs the map function on partial data and outputs the intermediate 〈key, value pairs in parallel. Then, the MapReduce-MPI framework communicates all the values with the same key across the distributed-memory system to the same process (i.e., shuffling phase). After the data shuffling, each process runs the reduce function and generates the final output.

Compared with Hadoop, MapReduce-MPI is more flexible when structuring computation: the programmer can specify any combination of map and reduce functions, including multiple map functions followed by one reduce, one map followed by multiple reduce functions, and multiple maps followed by multiple reduce functions. In Hadoop, on the other hand, iterations must be expressed 
as a chain of MapReduce jobs. In addition, MapReduce-MPI eliminates the use of the Hadoop Distributed File System (HDFS) for data input and output by using Lustre file system or the local disk of each node in the distributedmemory system directly. In other words, it eliminates the time-consuming datastaging phases required in Hadoop. Moreover, it runs on any platform that supports MPI and $\mathrm{C}++$, as do most distributed-memory systems including supercomputers. In comparison, Hadoop requires the installation of HDFS and Hadoop MapReduce components on the system. MapReduce-MPI also utilizes high-speed InfiniBand for data communication as a default.

Other software tools and libraries support the MapReduce programming model. In addition to Hadoop and MapReduce-MPI, newer libraries have been recently proposed such as Spark and Flink [24, 25]. Spark is a memory efficient, high performance and general purpose software system designed for computing clusters. It provides high-level APIs that support general work flows including MapReduce. Flink is another open source software system for distributed stream and batch data processing. Both software tools enable flexible data processing workflows and improve performance over Hadoop. None is currently featuring high performance computing properties that make them suitable for high-end clusters and supercomputers.

The MapReduce library in this paper is MapReduce-MPI because it supports the supercomputers used for our protein-docking simulations. Still the focus of

this paper is on the design of a general and scalable clustering method that fits into the general MapReduce programming model. Thus the algorithms for our analysis are library-agnostic and can be easily integrated into existing and new generations of MapReduce libraries as they are proposed.

\section{Related work}

This section discusses related work about clustering analysis of docking conformations and distributed clustering in MapReduce. 


\subsection{Clustering analysis of docking conformations}

Traditional clustering approaches group similar structural biology conformations through geometry-based clustering. Important work in this direction includes that of Lorenzen and Zhang [26], Bouvier et al. [27, Chang et al. [28], and Estrada et al. 4. Lorenzen and Zhang selected near-native docking conformations by assuming that a bigger cluster would be more likely to have better candidate conformations [26]. Bouvier et al. applied a Kohonen selforganizing map trained in a preliminary phase by using drug-protein contact descriptors [27. Chang et al. performed a simple cluster analysis for docking simulations and used the size of the clusters to estimate the vibrational entropy of the resulting conformations [28]. Estrada et al. identified near-native ligand conformations using a probabilistic hierarchical clustering and fuzzy c-means [4]. Such techniques require that data be stored in a centralized location in order to

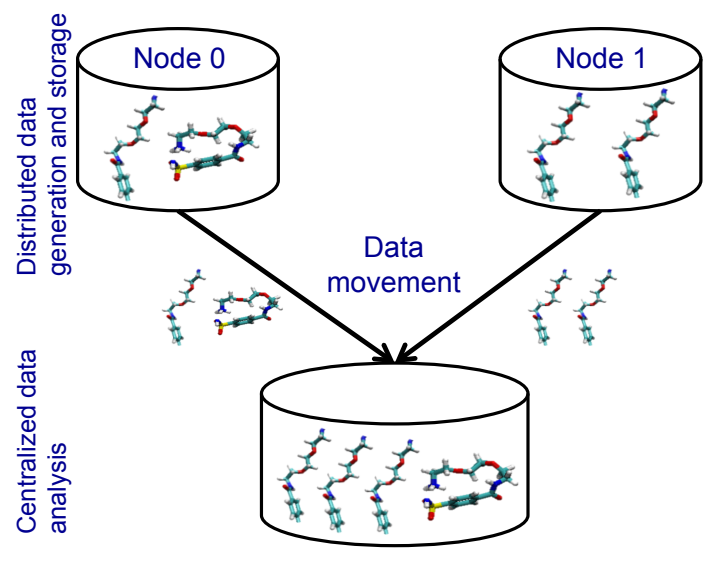

Figure 2: Traditional centralized comparison and clustering of large datasets generated on a distributed-memory system.

compute the RMSD of each ligand with respect to all the other ligands in the dataset. The analysis requires the molecular dataset to be moved in its entirety into a central server. Figure 2 shows an abstract representation of a centralized data analysis system in which all the ligands have to be moved to a local storage where they can be compared and clustered. The fully centralized approach is 
not scalable and can result in serious storage and bandwidth pressures on the server. Thus, the challenge involves ways to find these dense ligand clusters of geometric representations efficiently, especially when the data is acquired in a distributed way. While each conformation is small (on the order of 10 Kbytes), the number of conformations that must be moved across the distributed-memory system and compared is extremely large; depending on the type and number of proteins, the conformation dataset can comprise tens or hundreds of millions of ligands. This scenario is expected when thousands of processes perform individual docking simulations and store their results locally. As an example, consider the $\mathrm{D} @ \mathrm{H}$ project that is supported by more than 188,000 hosts. If all the hosts communicate their local results to a centralized server simultaneously, even when each host communicates data in terms of 100 ligand conformations (i.e., around $7 \mathrm{MB}$ ), the data sending across the distributed-memory system is more than 1 TB.

To avoid this big data movement among nodes, in previous work our group 15 proposed a distributed clustering method in MapReduce that first extracts relevant data properties locally and concurrently and then transforms the analysis problem (e.g., clustering or classification) into a search for property aggregates [6]. We briefly summarize the weak scalability of the method using up to 256 compute nodes of the Fusion cluster at Argonne National Laboratory and 2 TB datasets in [7. Our previous work considered only three-dimensional mappings and did not provide insights into the factors that affect performance scalability at large scale. In this paper we extend the previous work and explore six-dimensional data properties that result in better accuracy for the analysis. In addition, we perform an in-depth performance analysis to provide key in325 sights into how the large-scale performance is affected by the data content and convergence.

\subsection{Distributed clustering in MapReduce}

The MapReduce programming model has been used to analyze large data in science and engineering fields using clustering techniques. Some efforts have 
investigated well-known clustering methods such as k-means and hierarchical clustering, which were adapted to fit into the MapReduce framework [29, 30]. However, the resulting implementations suffer from the limitations of the clustering algorithms, which do not scale despite being formulated in MapReduce. A similar clustering approach based on the density of single points in an Ndimensional space was presented by Cordeiro et al. 31. The three algorithms presented in that paper rely on local clustering of subregions and merging of local results into a global solution (which can potentially suffer from accuracy issues), whereas our proposed approach considers the whole dataset and performs a single-pass analysis on it. Contrary to [31, when using the LG variation we no longer observe a correlation between space density and clustering efficiency.

Another group of research efforts performs a hashing step that partitions the input data into groups and then clusters each group in parallel. Important work includes that of Hefeeda et al. 32] and Rasheed and Rangwala 33. Hefeeda et al. designed an approximation algorithm that reduces the computation and memory overhead in kernel-based machine learning algorithms. The approximation algorithm uses locality sensitive hashing on the signature of each data record to hash close records in the same bucket. However, this method potentially suffers from accuracy issues when pursing performance by using a lower level of approximation. In our work, we deliver scalable performance without sacrificing the accuracy. Rasheed and Rangwala clustered metagenome sequence reads using a minwise hashing approach and agglomerative hierarchical clustering or greedy clustering. Their work requires computing the similarity of a given metagenome sequence read with groups of sequence reads, whereas in our work no comparison is needed between data records (i.e., ligand conformations).

355 In our work, scalability plays a key role and is particularly well supported by the LG variant. In [5], we used Hadoop and observed how the framework was a major hindrance to scalability. In [6, 7] and in this work, we move away from high-overhead frameworks that suffer from poor scalability on high-end clusters such as Hadoop, and we instead use MapReduce-MPI. In other words, by using MapReduce-MPI rather than Hadoop we move away from the overhead of 
the Hadoop Distributed File System (HDFS). HDFS acts as the central storage space for input and output data, adding additional space and operation time to move input and output data. In [6, 7] we reported our preliminary work on how to tune scalability by tuning the way metadata is handled. In the past work, we extended the algorithm searching for dense aggregation of metadata into two variations for fully distributed environments. In these two variations, data no longer needs to be moved a priori with HDFS, making our approach completely scalable. The two variations allow us to study the performance impact of exchanging extracted properties in contrast to exchanging property densities; this was not achievable in Hadoop because of the coarse-grained control on data placement of HDFS. In this paper we further extend the scalability study by presenting a larger set of performance results than those presented in [7.

\section{Methodology}

This section describes our method to capture relevant geometrical properties 375 in ligand conformations and search for densest metadata subspaces using the MapReduce programming model.

\subsection{Capturing relevant geometrical properties}

Our processing of docking simulation results requires extracting the geometrical shape (or property) of each docked ligand in the docking pocket of ${ }_{380}$ a protein. To this end, we perform a space reduction from the atom coordinates of the ligand conformation to a single point of three or six coordinates in a 3-D or 6-D space, respectively, depending on which reduction technique we are using. The three or six coordinates are the metadata that represents the ligand's geometry in the protein's docking pocket. Our approach relies on the key assumption that when geometrical properties are properly captured, ligand conformations with similar geometries are mapped into similar metadata. As Figure 3 shows close 3-D points in a 3-D mapping representing the geometry of similar ligand conformations. In other words, our space reduction should have 
the desired property of projecting ligands with a similar geometry closer into

the newly defined space, and the converged cluster with the highest density of points should include ligand conformations of interest for the pharmaceutical search. The empirical validation of this hypothesis is presented in Section 6 .

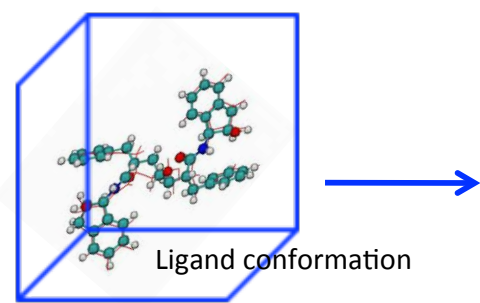

(a)

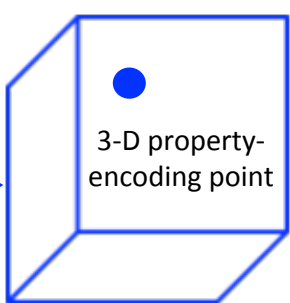

(b)

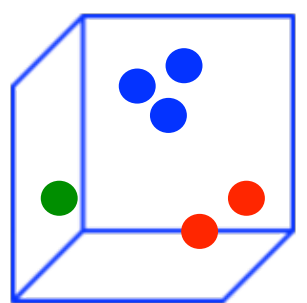

(c)

Figure 3: From the scientific data to extracted property: From the left to right a ligand conformation in the docking pocket of a protein, the point that encodes the geometrical property using either the 3-D or 3-Dlog mapping, and six points obtained in parallel that represent six ligand conformations clustered in three groups with three geometries.

We consider three variations of the mapping algorithm; all are based on projections and linear interpolations. The variations share the backbone reduction senting the conformation geometry. If the slope is negative, we use the negative logarithm of the absolute value. Contrary to the 3-D mapping variation, this 
variation better captures the geometrical properties of conformations that are tional analysis algorithms, such as k-means and fuzzy c-means clustering. These calculations may require moving conformations across nodes, thus causing many frequent communications and multiple storages of the same data across nodes. On the contrary, our space reduction can be applied individually and concur435 rently to each ligand conformation by transforming each molecule containing $p$ atomic coordinates in the three-dimensional space $(p \times 3)$ into a single point of $(1 \times 3)$ for the 3 -D and 3 -Dlog mappings and $(1 \times 6)$ for the 6 - $\mathrm{D}$ mapping, all in the Euclidean space. This transformation is performed locally on the compute node that generates and stores the ligand conformation, and thus no commu- 


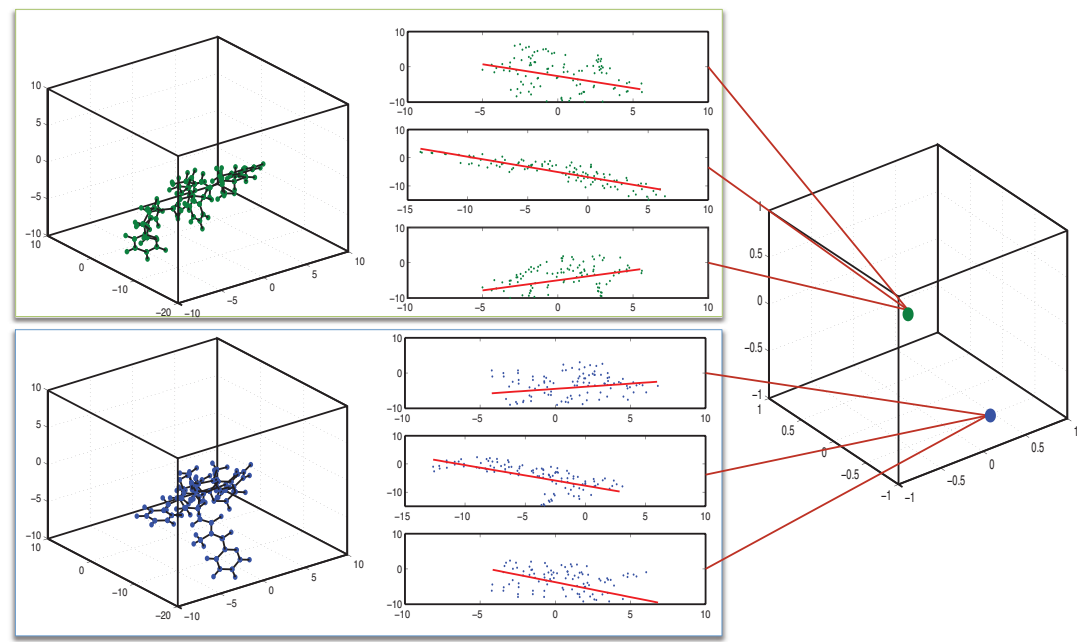

Figure 4: Capturing relevant geometrical properties by using projection and linear interpolation for the 3-D mapping variation.

nication is required during this phase. The projections and interpolations are low-cost processes in terms of computing and memory requirements.

\subsection{Searching for densest metadata subspaces}

After mapping ligands' geometrical properties into property-encoding metadata (i.e., three-dimensional points for the 3-D and 3-Dlog mappings or sixdimensional points for the 6-D mapping) rather than dealing with raw atom coordinates, we build an N-D tree by recursively partitioning the N-D space into fixed-sized subspaces, each of which form the tree nodes. By doing so, we implicitly transform the analysis problem from a clustering or classification problem into a search of the smaller subspaces in the newly defined metadata space (i.e., an octant for the 3-D and 3-Dlog mappings or a 6-D space for the 6-D mapping) with high property aggregates.

From the data structure point of view, we reshape the space of propertyencoding points into an N-D tree and search for the deepest, densest tree nodes (i.e., the nodes in the deepest level of the N-D tree that contain a minimum number of points). These nodes contain the solution to our analysis problem. 
Our search for dense tree nodes (or properties) begins with each compute node generating its N-D tree on its own local data by recursively subdividing the space into $2^{k}$ subspaces: $8\left(2^{3}\right)$ subspaces for the 3 -D and 3-Dlog mapping and $64\left(2^{6}\right)$ subspaces for the 6-D mapping. Figure 5 shows an example of a dataset of $1 \mathrm{hbv}$ ligand conformations when docked in the HIV protease. Figure 5.a shows the docking pocket for the protein with a docked ligand. Figure 5 b is the result of the 3-D mapping, after the mapping of $1 \mathrm{hbv}$ ligand confirmations into metadata has been performed. The compute nodes build an octree by assigning octkeys to the points, an N-D tree by assigning N-D keys to the points in the case of 6-D mapping. A point belongs to a specific tree node based on its key. The point's key is generated as follows. We initially determine the edge size (i.e., $\mathrm{N}-\mathrm{D}$ resolution) of the N-D space containing all the projected conformations. Since we are dealing with the 3-D mapping in this example, we divide the initial space into eight subspaces of the same size, half the original edge size. A similar process is followed for the 3-Dlog mapping; however, when using the 6-D mapping, the metadata space is subdivided into 64 subspaces (not shown here). Every subspace is given a unique identifier ranging from 0 to 7 for the 3 -D space or from 0 to 63 for the 6 -D space, based on its position in the N-D space. The key of each point is extended by attaching the subspace identifier to the point's 475 key by padding the left side with the identifier. This process is recursively repeated an arbitrary number of times on each subspace to produce a complete key for each point (key[1...Nkey]), where $N k e y$ is the number of digits selected to represent each point. As previously observed in [11, Nkey can be empirically defined, and a value key of 15 digits is sufficient to capture diverse geometries in the dataset of ligand conformations considered in this work. Figure 5 c shows an example of the generated octree for the 3-D mapping points in Figure 5.b.

Alternative approaches to octkeys that can be used to map data points to octree spaces include the Z-order curve (or Morton order) and the Hilbert curve [34, 35. Both methods map multidimensional data to one dimension. ${ }_{485}$ Take the Morton order for example, the Morton code for a multidimensional point is calculated by interleaving the binary representations of the point's co- 


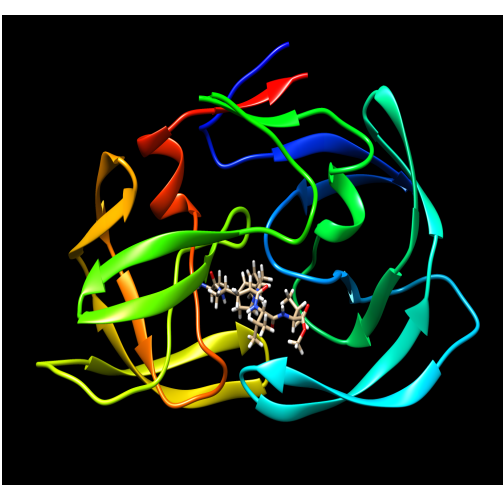

(a) 1hbv ligand in protein

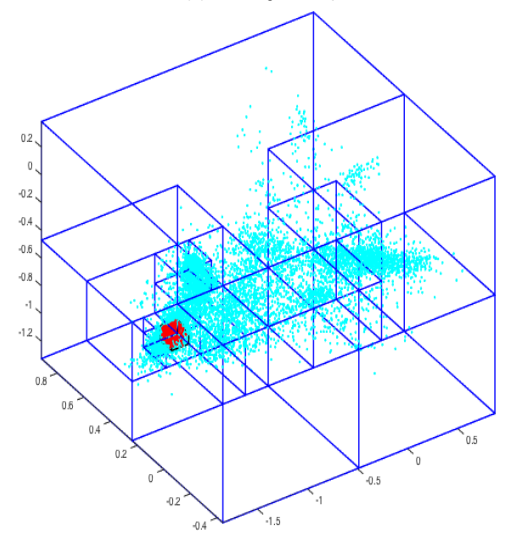

(c) Octree
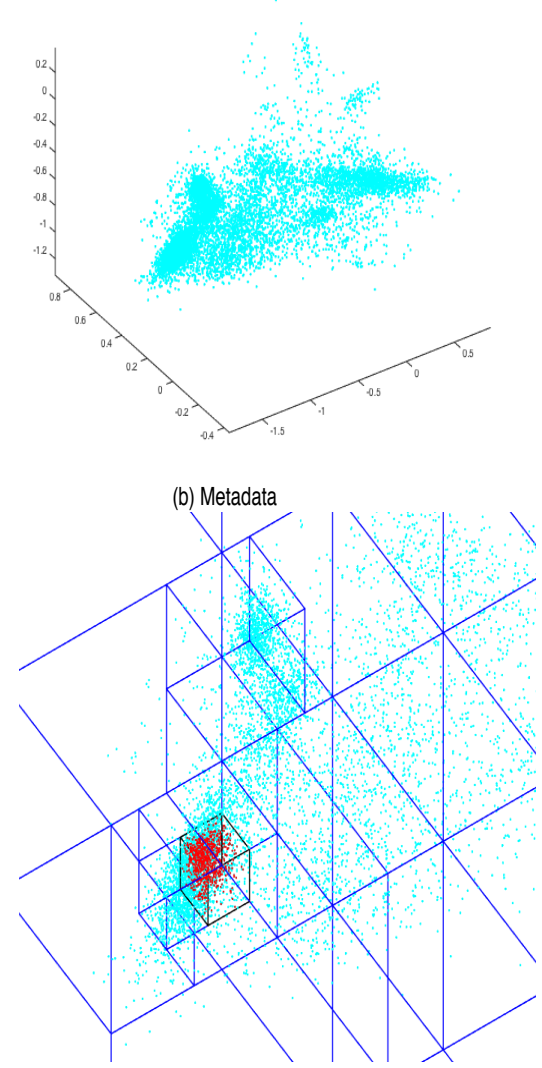

(d) Densest octant

Figure 5: Example of a metadata space of 3-D points generated from a dataset of ligand conformations and its octree built to identify the densest octant.

ordinate values in all dimension. For example, given a two dimensional data point $\langle 2,5\rangle$, in which each decimal value is represented by 8 bits in its binary format, we can calculate the Morton code for this data point. The binary representation of decimal value 2 is 00000010 , and the binary representation of decimal value 5 is 00000101 , the resulting Morton code for $\langle 2,5\rangle$ is 0000000000100110, which is obtained by interleaving the binary representation of 5 and 2. This Morton code can be used to build octrees. The general process is to compute the Morton code for every multidimensional data point in the dataset, then sort the Morton codes. Once sorted, the Morton order ensures 
good spatial locality since close multidimensional points tend to be close in the Morton order. Hilbert curve is based on a similar idea, but use a different mapping method to map the multidimensional point into one dimension. These methods bring good spatial locality, but only when the entire dataset is sorted 
distributed-memory system. Thus, the next challenge we face is how to efficiently explore certain level of the tree, which is to count the aggregates of close property-encoding points of the tree nodes (i.e. subspaces) on a specific level, in a distributed way. We address the challenge by defining an effective search algorithm based on an N-D tree search. The search algorithm counts scalar property aggregates representing locally stored metadata densities and identifies the densest tree nodes in the N-D tree (i.e., the deepest nodes with a minimum number of metadata items or aggregates). We design two general variations of the search algorithm that we call GlobalToLocal (GL) and LocalToGlobal (LG). The two variations are depicted in Figure 6 for a simplified case study that maps the conformations into a 2-D space. The two algorithms can be implemented for different MapReduce APIs such as Spark and Hadoop or distributed parallel programming libraries such as MPI. In this work, we implemented our variations in MapReduce-MPI because the library supports the supercomputers on which the simulations we used for the data generated were executed.
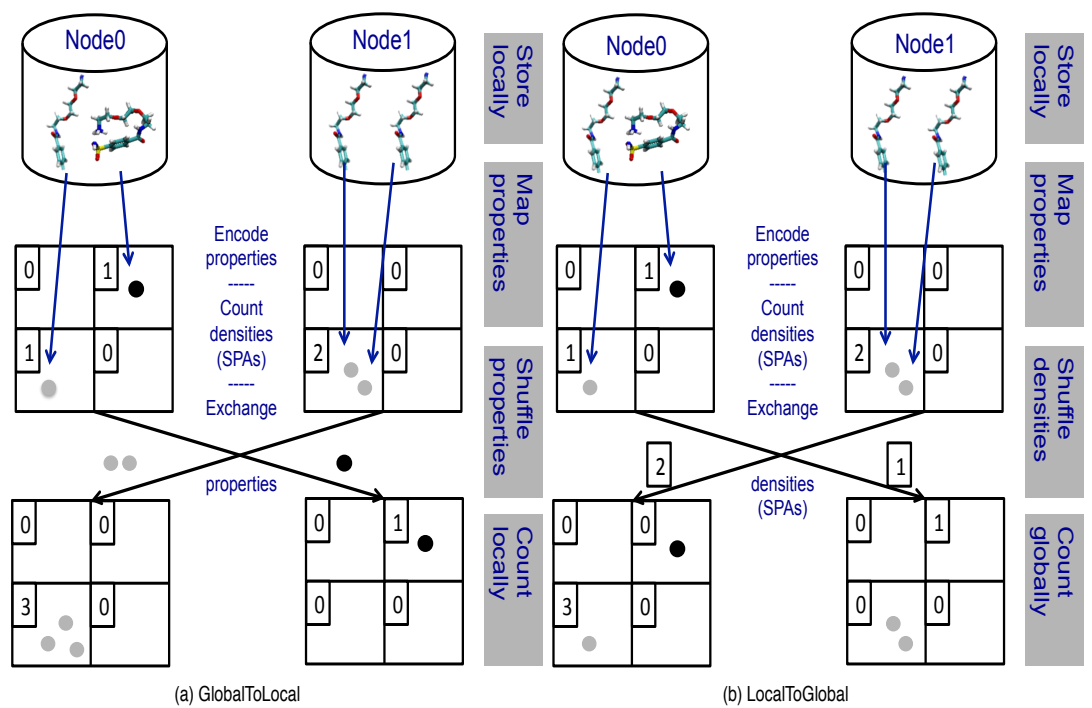

Figure 6: Examples of exchange of properties in the GlobalToLocal variation and exchange of scalar property aggregations in the LocalToGlobal variation. 
In GlobalToLocal, each compute node has an assigned subspace of the N-D space, and the extracted properties (i.e., the 3 -D or 6 -D points) are communicated among compute nodes so that each one has all the information needed to analyze similar properties in the assigned subspace. The analysis is applied to each compute node's local properties iteratively. This process rebuilds a global view of the information content in the scientific data so that similar points representing similar geometries eventually reside on the same compute node or nodes that are topologically close to each other (Figure 6.a). The atom coordinates of the ligands generating the property-encoding points are not moved from their original compute nodes. Property densities are then iteratively counted locally while searching for convergence.

In LocalToGlobal, after capturing relevant properties, each compute node applies the data analysis operation to its local extracted properties and computes scalar property aggregates based on densities for the entire assigned N-D space. In this scenario, each compute node has a disjointed view of the entire N-D space containing the metadata for the ligand conformations stored on its disk. The union of the disjointed N-D spaces is important for the knowledge acquisition of the densest subspaces. This is pursued in the variation of the search algorithm through the communication of the local aggregates among compute nodes so that each compute node has all the partial results necessary to analyze the density globally. An aggregate operation (i.e., sum) is applied to the partial results. Communication of properties and aggregates is done iteratively. ${ }_{565}$ Communication in GlobalToLocal happens only once, and the communicated package is larger. On the other hand, communication in LocalToGlobal happens multiple times, and each time the communicated package is smaller.

In LocalToGlobal, instead of exchanging extracted metadata, compute nodes exchange partial densities represented as scalar property aggregates (Figure 6(b)). Each compute node preserves a global vision of the metadata associated with its local data and counts its densities before shuffling the densities (or aggregates) rather than the metadata with other compute nodes. After shuffling the aggregates, each compute node sums the aggregates to obtain the global cluster 
densities while searching for convergence. Schemes and optimizations similar to variation used (GL or LG). When exploring the tree nodes on a specific level of the N-D tree, we need to exchange property encoding points and compute densities for the tree nodes at this level (in GL) or compute partial densities for 
the tree nodes at this level and exchange scalar property aggregates (in LG). The communication size and pattern for each level are different. Let's consider the following examples, at the root level, we need to count the density of the root node (i.e., the entire N-D space). In the GL variation, all the property encoding points are communicated to one node, and one process computes the density of the root node. In the LG variation, all the processes computes partial density of the root node, then one scalar property aggregate that describes the partial density of the root node is sent from every process to one process, which computes the density of the root node. Consider the middle level of the tree, level 8 in our example, in the GL variation, all the property encoding points with the same first 8 digits in their octkey are communicated to the same node, and the process computes the density of this subspace (i.e., tree node). In the LG variation, each process computes the partial densities of the subspaces starting with the same first 8 digits, and the scalar property aggregates of the subspaces are communicated to appropriate processes for density computation. Generally, when moving down the tree, there exist more tree nodes (i.e., more subspaces) for which we need to compute the densities. As a result, the load imbalance in communication and computation in the GL variation and the communication size in the LG variation increase. Hence, the processing time increase, as we will further discuss in Section 5.5. For the same of efficiency, in our work we explore the tree levels in a binary search way. This binary search comes with two key benefits. First, we reduce the number of tree levels that we need to explore. Second, we avoid exploring deep levels of the tree as the bottom-up method for example does.

\subsection{Integration into MapReduce-MPI}

The MapReduce programming model naturally accommodates the capturing of properties from local data and the iterative search for either properties or densities in its map and reduce functions, respectively. Thus, we integrated our two variations (i.e., the GlobalToLocal and the LocalToGlobal) into the MapReduce-MPI framework rather than implementing a new MPI-based frame- 
work from scratch.

In the GlobalToLocal variation, the operation of capturing relevant properties is implemented as the map function. It takes the identifier and coordinates of each ligand conformation as the input key and value, respectively; applies the geometry reduction operation; and outputs the id and the propertyencoding point of each conformation as the intermediate key and value pair. The MapReduce-MPI library shuffles the property-encoding points across the distributed-memory system to rebuild the global knowledge of the N-D space on each node. It achieves this goal by communicating all the property-encoding points in one N-D subspace to one process such that this process has all the information needed to explore the N-D tree locally. Then, the operation of counting property densities is implemented as the reduce function. This function takes as input the id of the tree node and all the property-encoding points in the node and iteratively explores its local N-D tree by counting the density of the nodes in one level of the N-D tree until the deepest and densest tree node is found.

The LocalToGlobal variation has two map functions. The first function captures the relevant geometrical properties the same way as in the GlobalToLocal variation. After the relevant properties are extracted by the first map function, the second map function counts locally the property aggregates for a certain level of the N-D tree nodes. It takes the id and the property-encoding point of each conformation as the input key and value pair, respectively; counts the aggregates for tree nodes at a certain level; and outputs the id and aggregates of each tree node as the intermediate key and value pairs. The exploration of the N-D tree starts at the middle level of the tree and branches up or down depending on whether a dense enough node is found. The MapReduce-MPI framework shuffles the id and all the aggregates of each node across the distributed-memory system. Then, the reduce function applies a sum operation to all the aggregates to compute the density of the nodes. The process of counting aggregates (i.e., the second map function) and summing aggregates (i.e., the reduce function) is iterated until the deepest and densest node is found. 
The differences between the two variations lie in the communication phase (i.e., data shuffling). In the GlobalToLocal variation communication happens only once, and the size of the communicated data (i.e., the property-encoding points) is larger. In comparison, in the LocalToGlobal variation the communication happens multiple times, and each time the size of the communicated data (i.e., local aggregates) is smaller.

\section{Performance}

In this section we present performance results at a smaller scale in terms of comparisons across searches and at a larger scale in terms of weak scalability.

\subsection{Platforms}

We consider two platforms with two different scales in terms of number of nodes. At the small scale, we use 64 of the Gordon's compute nodes. Gordon, a Track 2 supercomputer at the San Diego Supercomputer Center, features powerful flash memory storage private to each compute node that is used as a representative of a fully distributed-memory system [37. For the tests, we were limited to 64 nodes only, because of allocation constraints set by the provider. Each node contains two 8-core 2.6 GHz Intel EM64T Xeon E5 (Sandy Bridge) processors and $64 \mathrm{~GB}$ of DDR3-1333 memory and mounts a single $300 \mathrm{~GB}$ SSD. The nodes are connected by a $4 \times 4 \times 4$ D torus with adjacent switches connected by three $4 \times$ QDR InfiniBand links.

At the large scale, we use 256 compute nodes of Fusion, a 320-node computing cluster at the Laboratory Computing Resource Center at Argonne National Laboratory. Each of Fusion's compute nodes contains two Nehalem $2.6 \mathrm{GHz}$ dual-socket, quad-core Pentium Xeon processors and 36 GB of RAM and 250 GB local disk. The nodes are connected by InfiniBand QDR at $4 \mathrm{~GB} / \mathrm{s}$ per link.

\subsection{Data distributions}

The information embedded in the dataset of ligand conformations can have different contents and meanings, resulting in different logical and physical meta- 
data distributions. For instance, the collected ligand conformations may more or less strongly converge toward a single similar geometry or may not converge at all. To capture different types and degrees of convergence as well as their impact in the communication, we consider five metadata ensembles generated by synthetically sampling ligand conformations from real Docking@Home data. In each ensemble the conformations are selected to fit specific property distributions in the 3-D and 6-D metadata spaces (logical distribution). Figure 7 shows the five ensembles for the 3-D and 3-Dlog mapping. The 6-D mapping is not presented here because of the complexity of its representation, but similar conclusions hold for it. The five ensembles reflect five scientific conclusions in terms of convergence toward specific ligand geometries. (Note that in the following section, we discuss the performance in the context of 3-D mapping. Hence the N-D search is performed as an octree search in 3-D space.) Similar behaviors are observed for 3-Dlog and 6-D mappings in terms of performance and are omitted here. The impact of different mapping methods lies in accuracy.

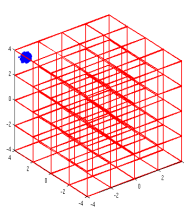

(a) $1 \mathrm{D}$

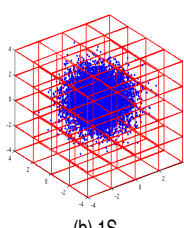

(b) $1 \mathrm{~S}$

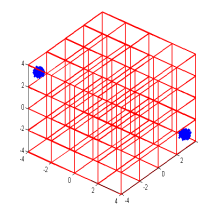

(c) $2 D$

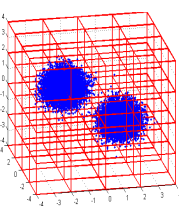

(d) $2 S$

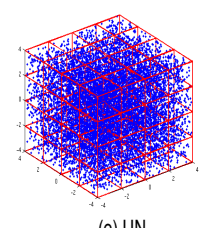

(e) UN

Figure 7: Five scenarios of logical distributions of data: 1D, 1S, 2D, 2S, and UN.

In the first scenario with one dense cluster (1D), the information content

3 -D points densely populate one small region of the metadata space while most of the remaining space is empty, as shown in Figure 7.a. We select ligands from the Docking@Home dataset whose geometries generate 3-D points with normal distribution around one point in the 3-D space with a standard deviation the information content in the data more loosely converges toward one ligand conformation; the 3-D points sparsely populate one larger region of the space, 
as shown in Figure 7.b. The ligand geometries generate points with normal distribution around one point in the 3 -D space with a standard deviation of

most likely in a semi-decentralized distribution in which points mapping close properties are collected by the same node or topologically close nodes; hence, there is uniformity of the property-encoding points inside the nodes' storages. 
In round-robin distributions, the conformations and their points that belong to the same subspace in the logical distribution are stored in separate physical storage in a round-robin manner. This scenario happens most likely in a fully decentralized, synchronous distribution in which points are collected at each predefined time interval; hence, the data points for each time interval are stored in separate storage across the distributed system. In random distributions, the conformations and their points are randomly stored in the physical storage of all the system nodes. This scenario simulates the fully decentralized asynchronous manner. In each scenario, the whole dataset is roughly evenly distributed among the physical storage sites of the testing machine.

\subsection{Datasets}

For testing, we build two synthetic datasets, each including the five logical distributions. We use the smaller dataset of up to 100 million ligands (250 GB) on up to 64 nodes of Gordon to compare the GlobalToLocal versus the LocalToGlobal variations. We use the larger dataset of up to 800 million ligands (2TB) on up to 256 nodes of Fusion to study the scalability of the LocalToGlobal variation. For the smaller dataset, we consider all three physical distributions (i.e., uniform, round-robin, and random); for the larger dataset, we use only the round-robin distribution. In all tests we transform each ligand conformation into a single point (metadata); the point location in the N-D tree is defined by a 15-digit key.

\subsection{Small scale: comparison across searches}

We compare and contrast the GlobalToLocal versus LocalToGlobal variations to assess their sensitivity to the logical and physical distributions of the dataset. The test is performed on Gordon using the smaller dataset and up to 64 nodes available as part of an XSEDE allocation. We measure and present the total execution time of the two variations on fifteen data distributions (i.e., five logical distributions and three physical distributions). Figure 8 shows the total time in seconds (aggregated across all processes) of GlobalToLocal (GL) 
and LocalToGlobal (LG) for the five logical distributions (i.e., one dense cluster 1D, one sparse cluster 1S, uniform UN, two dense clusters 2D, and two sparse clusters $2 \mathrm{~S}$ ) and the round-robin physical distribution. Each time presented here is the summation of the execution times of all processes in each run. The wall time of the program can be calculated by dividing the total execution time by the number of processes since it is a MPI programming model. Similar results were observed in the uniform and random physical distributions. All the results 785 are included in the discussion below, and the complete set of times is reported in Table 1 


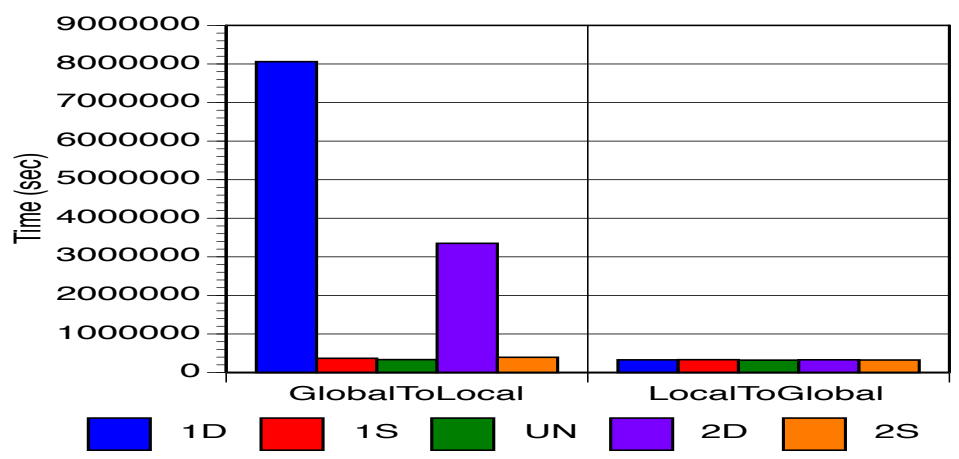

(a) Uniform

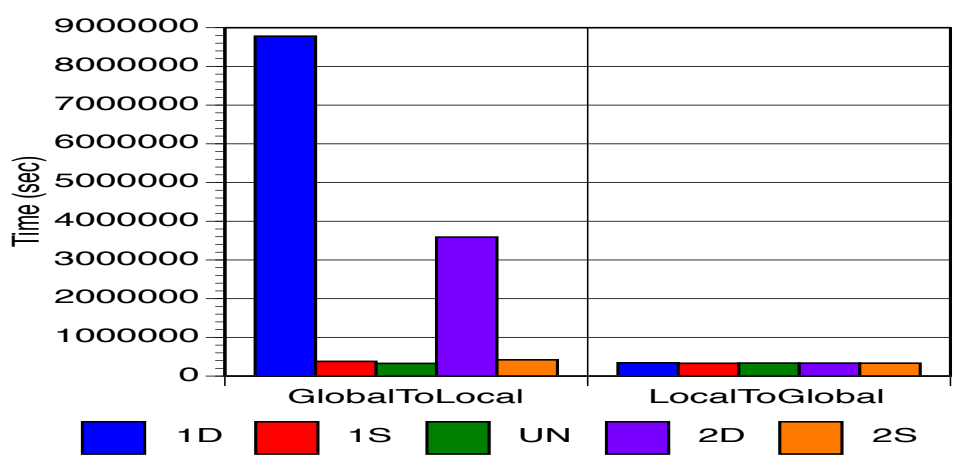

(b) Round-robin

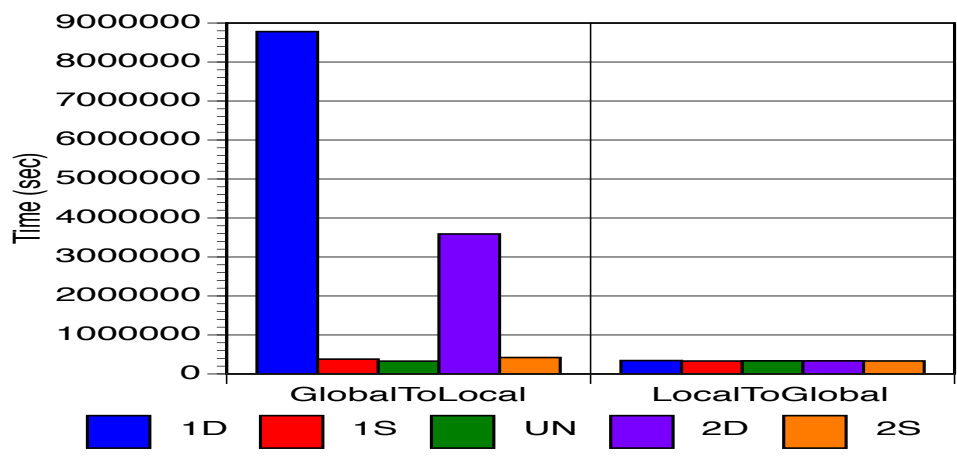

(c) Random

Figure 8: Total execution time for GlobalToLocal and LocalToGlobal on the five logical distributions and the uniform, round-robin, and random physical distributions. 
Table 1: Total times in seconds across processes broken down into distinctive components: Map (M), Shuffling (S), Overhead (O), Reduce (R), and Total $(\mathrm{T})$.

\begin{tabular}{|c|c|c|c|c|c|c|c|c|c|c|}
\hline \multicolumn{11}{|c|}{ Uniform } \\
\hline & \multicolumn{2}{|c|}{$1 \mathrm{D}$} & \multicolumn{2}{|c|}{$1 \mathrm{~S}$} & \multicolumn{2}{|c|}{ UN } & \multicolumn{2}{|c|}{$2 \mathrm{D}$} & \multicolumn{2}{|c|}{$2 \mathrm{~S}$} \\
\hline & GL & LG & GL & LG & GL & LG & GL & LG & GL & LG \\
\hline $\mathrm{M}$ & $2.64 \mathrm{E}+05$ & $2.68 \mathrm{E}+05$ & $2.65 \mathrm{E}+05$ & $2.68 \mathrm{E}+05$ & $2.65 \mathrm{E}+05$ & $2.68 \mathrm{E}+05$ & $2.65 \mathrm{E}+05$ & $2.68 \mathrm{E}+05$ & $2.64 \mathrm{E}+05$ & $2.69 \mathrm{E}+05$ \\
\hline $\mathrm{S}$ & $2.35 \mathrm{E}+04$ & $2.65 \mathrm{E}+03$ & $1.40 \mathrm{E}+03$ & $2.74 \mathrm{E}+03$ & $1.32 \mathrm{E}+03$ & $1.82 \mathrm{E}+02$ & $9.88 \mathrm{E}+03$ & $2.71 \mathrm{E}+03$ & $2.63 \mathrm{E}+03$ & $1.56 \mathrm{E}+03$ \\
\hline $\mathrm{O}$ & $7.77 \mathrm{E}+06$ & $5.86 \mathrm{E}+04$ & $1.01 \mathrm{E}+05$ & $6.18 \mathrm{E}+04$ & $6.69 \mathrm{E}+04$ & $5.39 \mathrm{E}+04$ & $3.07 \mathrm{E}+06$ & $5.91 \mathrm{E}+04$ & $1.26 \mathrm{E}+05$ & $5.45 \mathrm{E}+04$ \\
\hline $\mathrm{R}$ & $8.63 \mathrm{E}+03$ & $6.95 \mathrm{E}+00$ & $1.64 \mathrm{E}+03$ & $8.38 \mathrm{E}+00$ & $1.72 \mathrm{E}+03$ & $1.32 \mathrm{E}+00$ & $6.50 \mathrm{E}+03$ & $7.98 \mathrm{E}+00$ & $2.06 \mathrm{E}+03$ & $6.43 \mathrm{E}+00$ \\
\hline $\mathrm{T}$ & $8.06 \mathrm{E}+06$ & $3.29 \mathrm{E}+05$ & $3.69 \mathrm{E}+05$ & $3.33 \mathrm{E}+05$ & $3.35 \mathrm{E}+05$ & $3.22 \mathrm{E}+05$ & $3.35 \mathrm{E}+06$ & $3.30 \mathrm{E}+05$ & $3.95 \mathrm{E}+05$ & $3.25 \mathrm{E}+05$ \\
\hline \multicolumn{11}{|c|}{ Round-Robin } \\
\hline & \multicolumn{2}{|c|}{$1 \mathrm{D}$} & \multicolumn{2}{|c|}{$1 \mathrm{~S}$} & \multicolumn{2}{|c|}{$\mathrm{UN}$} & \multicolumn{2}{|c|}{$2 \mathrm{D}$} & \multicolumn{2}{|c|}{$2 \mathrm{~S}$} \\
\hline & GL & LG & GL & LG & GL & LG & GL & LG & GL & LG \\
\hline M & $2.64 \mathrm{E}+05$ & $2.69 \mathrm{E}+05$ & $2.64 \mathrm{E}+05$ & $2.70 \mathrm{E}+05$ & $2.65 \mathrm{E}+05$ & $2.68 \mathrm{E}+05$ & $2.64 \mathrm{E}+05$ & $2.69 \mathrm{E}+05$ & $2.65 \mathrm{E}+05$ & $2.68 \mathrm{E}+05$ \\
\hline $\mathrm{S}$ & $1.91 \mathrm{E}+04$ & $3.65 \mathrm{E}+03$ & $1.33 \mathrm{E}+03$ & $5.04 \mathrm{E}+03$ & $1.47 \mathrm{E}+03$ & $2.50 \mathrm{E}+03$ & $1.04 \mathrm{E}+04$ & $3.85 \mathrm{E}+03$ & $2.68 \mathrm{E}+03$ & $3.98 \mathrm{E}+03$ \\
\hline $\mathrm{O}$ & $8.49 \mathrm{E}+06$ & $7.10 \mathrm{E}+04$ & $1.13 \mathrm{E}+05$ & $5.62 \mathrm{E}+04$ & $6.02 \mathrm{E}+04$ & $6.55 \mathrm{E}+04$ & $3.30 \mathrm{E}+06$ & $6.18 \mathrm{E}+04$ & $1.51 \mathrm{E}+05$ & $6.04 \mathrm{E}+04$ \\
\hline $\mathrm{R}$ & $9.35 \mathrm{E}+03$ & $1.06 \mathrm{E}+01$ & $1.90 \mathrm{E}+03$ & $1.31 \mathrm{E}+01$ & $1.93 \mathrm{E}+03$ & $6.16 \mathrm{E}+00$ & $7.09 \mathrm{E}+03$ & $1.11 \mathrm{E}+01$ & $2.56 \mathrm{E}+03$ & $1.13 \mathrm{E}+01$ \\
\hline $\mathrm{T}$ & $8.78 \mathrm{E}+06$ & $3.43 \mathrm{E}+05$ & $3.80 \mathrm{E}+05$ & $3.31 \mathrm{E}+05$ & $3.28 \mathrm{E}+05$ & $3.36 \mathrm{E}+05$ & $3.59 \mathrm{E}+06$ & $3.35 \mathrm{E}+05$ & $4.21 \mathrm{E}+05$ & $3.33 \mathrm{E}+05$ \\
\hline \multicolumn{11}{|c|}{ Random } \\
\hline & \multicolumn{2}{|c|}{$1 \mathrm{D}$} & \multicolumn{2}{|c|}{$1 \mathrm{~S}$} & \multicolumn{2}{|c|}{$\mathrm{UN}$} & \multicolumn{2}{|c|}{$2 \mathrm{D}$} & \multicolumn{2}{|c|}{$2 \mathrm{~S}$} \\
\hline & GL & LG & GL & LG & GL & LG & GL & LG & GL & LG \\
\hline $\mathrm{M}$ & $2.66 \mathrm{E}+05$ & $2.69 \mathrm{E}+05$ & $2.65 \mathrm{E}+05$ & $2.69 \mathrm{E}+05$ & $2.65 \mathrm{E}+05$ & $2.69 \mathrm{E}+05$ & $2.66 \mathrm{E}+05$ & $2.69 \mathrm{E}+05$ & $2.64 \mathrm{E}+05$ & $2.69 \mathrm{E}+05$ \\
\hline S & $1.94 \mathrm{E}+04$ & $3.73 \mathrm{E}+03$ & $1.35 \mathrm{E}+03$ & $4.92 \mathrm{E}+03$ & $1.25 \mathrm{E}+03$ & $2.53 \mathrm{E}+03$ & $1.02 \mathrm{E}+04$ & $3.77 \mathrm{E}+03$ & $3.17 \mathrm{E}+03$ & $3.98 \mathrm{E}+03$ \\
\hline $\mathrm{O}$ & $8.63 \mathrm{E}+06$ & $6.16 \mathrm{E}+04$ & $1.14 \mathrm{E}+05$ & $5.72 \mathrm{E}+04$ & $6.09 \mathrm{E}+04$ & $6.28 \mathrm{E}+04$ & $3.62 \mathrm{E}+06$ & $5.67 \mathrm{E}+04$ & $1.66 \mathrm{E}+05$ & $6.28 \mathrm{E}+04$ \\
\hline $\mathrm{R}$ & $9.52 \mathrm{E}+03$ & $1.06 \mathrm{E}+01$ & $2.03 \mathrm{E}+03$ & $1.30 \mathrm{E}+01$ & $1.96 \mathrm{E}+03$ & $6.12 \mathrm{E}+00$ & $7.81 \mathrm{E}+03$ & $1.09 \mathrm{E}+01$ & $2.99 \mathrm{E}+03$ & $1.07 \mathrm{E}+01$ \\
\hline $\mathrm{T}$ & $8.93 \mathrm{E}+06$ & $3.35 \mathrm{E}+05$ & $3.82 \mathrm{E}+05$ & $3.32 \mathrm{E}+05$ & $3.29 \mathrm{E}+05$ & $3.34 \mathrm{E}+05$ & $3.90 \mathrm{E}+06$ & $3.30 \mathrm{E}+05$ & $4.37 \mathrm{E}+05$ & $3.36 \mathrm{E}+05$ \\
\hline
\end{tabular}


When looking at the execution times across the five logical distributions for both the GlobalToLocal and LocalToGlobal variations, we observe that for all three physical distributions, the performance of the GlobalToLocal variation is

library either for synchronizing processes at the end of each MapReduce step (i.e., the implicit MPI_Allreduce operations to communicate small bookkeeping 
information such as the total number of $\langle$ key, value $\rangle$ pairs processed by the map or reduce function) or for awaiting certain processes to complete their Map or Reduce in the case of load imbalance. The reduce time is the time to aggregate properties in GlobalToLocal or the time to aggregate densities in LocalToGlobal. In the figure, we compute the percentages using the average execution time in seconds over three runs; the time traces are obtained by using TAU [38. We instrumented the source code so that only a limited number of events (i.e., the time to perform the key map, data shuffling, and reduce functions in the two variations) are measured by TAU; thus, the overhead introduced by TAU is negligible.

When looking at the percentages of time for each variation across the five logical distributions, we observe that for all three physical distributions, the overhead percentage for the GlobalToLocal varies significantly across the five logical distributions, while the overhead percentage for the LocalToGlobal is relatively constant. To be more specific, in the round-robin physical distribution, when the information content in the dataset strongly converges to one cluster or two clusters (i.e., the 1D and 2D logical distribution), the overhead for GlobalToLocal is $96.4 \%$ and $91.6 \%$, respectively. When the information content loosely converges to one cluster or two clusters (i.e., the $1 \mathrm{~S}$ and $2 \mathrm{~S}$ logical distribution), the overhead is $27.4 \%$ and $31.9 \%$, respectively. In the UN logical distribution, when the information content does not converge at all, the overhead is at minimum 20.0\%. In comparison, for the LocalToGlobal variation, 840 the overhead is consistently around $19.0 \%$ across all five logical distributions. In general, we observe that when the datasets contain strong scientific conclusions (i.e., the 1D and 2D logical distributions), GlobalToLocal has a significant overhead, while LocalToGlobal has consistent overhead across the five logical distributions. The reason is that in the one dense cluster 1D and two dense ${ }_{845}$ cluster $2 \mathrm{D}$ scenarios, the properties (3-D points) are densely populated in a small octant space; thus, in order to explore the octree, GlobalToLocal requires relocating all the properties (3-D points) to one or two processes, respectively, on which the iterative search is performed locally. When points are sparsely 
distributed (one sparse cluster 1S and two sparse cluster 2S), GlobalToLocal that with the round-robin and random physical distributions.

To better understand the reasons behind the observed overheads, in Figure 10 we present the typical time pattern per process for the uniform, round- 
robin, and random physical distributions and the five logical distributions; each bar is a collection of 1,024 thin bars, one per process. For each subfigure, each process time in the two bars is normalized with respect to the longest process time for both GlobalToLocal and LocalToGlobal. This approach allows us to better compare the two variations. Figure 10 shows that overheads have two components: (1) a much larger component that is present in GlobalToLocal only and is due to load imbalance and (2) a smaller component that is present in both variations and is due to a collective communication embedded into MapReduceMPI when synchronizing all the processes. This is visible in the figure where thin black lines (corresponding to the Reduce phase) at the top of the bars are observed in one dense cluster $1 \mathrm{D}$ and two dense clusters $2 \mathrm{D}$ for one and two processes, respectively, that perform most of the reduce task (i.e., the aggregate operation on the extracted properties). During this time, the remaining processes sit idle until the Reduce step is completed. For the one sparse cluster 1S and two sparse clusters 2S scenarios and GlobalToLocal, we still observe load imbalance (in the form of drifting lines at the top of the bars) but in a smaller proportion compared with one dense cluster 1D and two dense clusters 2D. In this case, multiple processes across multiple nodes perform the aggregation operation on more dispersed properties. The synchronization time for the 1,024 processes is similar for the two variations; it is negligible compared with the other overhead component in GlobalToLocal and can take up to $20 \%$ of the total time in LocalToGlobal. Both Figures 9 and 10 convey the important findings that LocalToGlobal is able to perform implicit load balancing regardless of the logical distributions and physical data distributions.

\subsection{Large scale: weak-scalability study}

We focus on the LocalToGlobal variation because it exhibits constant performance at the small scale independent of the logical and physical distributions of the metadata [6]. We compare and contrast the weak scalability of this search variation on Fusion when the data size increases from 128 GB (50 million ligands) to 2 TB (800 million ligands) while the number of nodes increases from 
MPI process per core and a fixed data size per core of 1 GB. In our tests, we consider the five different logical distributions (i.e., 1D, 1S, 2D, 2S, UN).

We divide the total execution time in map, data shuffling, and reduce times. Each test is repeated three times; the measurements reported are average values. 915 Figure 11 shows the results of the scalability study. Specifically, the figure shows the average runtime in seconds and its variation across the three runs when using 16 nodes ( 128 cores), 32 nodes ( 256 cores), 64 nodes (512 cores), 128 nodes (1,024 cores), and 256 nodes (2,048 cores). We observe that as the number of nodes increases, the map and reduce times stay constant while the map and reduce times are computation times. When studying weak scaling, the computation work per node is fixed; thus, we observe a constant behavior. However, the data shuffling times are associated with communication times during which either metadata or property aggregates are moved across nodes. size of data and the number of processes increase, the communication overhead increases accordingly.

At small scale (i.e., on 16 nodes on Fusion), the execution times do not vary across the different logical metadata distributions; this result is consistent with what we observed on Gordon when using its 64 nodes. At large scale (i.e., when using 256 nodes), however, the execution times (mainly shuffling times) vary with the logical distribution. Specifically, dense metadata (i.e., 1D and 2D) has smaller execution times (and associated shuffling times), whereas sparse metadata (i.e., 1S, 2S, and UN) has larger execution times (and associated on Gordon or Fusion with only 16 nodes.

To investigate what causes the runtime variance, we look at the time breakdown of the data shuffling. Based on empirical observation in our previous work 11, we represent each point with a key that is 15 digits long (Nkey =15). ${ }_{940}$ Consequently, our search along the tree nodes explores four levels of the tree 
and performs four shuffling calls, as described in detail in Section 4.2. Table 2 shows the four levels explored in our search for the five logical distributions.

Table 2: Four levels explored in our tree search for the five logical distributions when representing each point with a 15-digit key.

\begin{tabular}{|l|r|r|r|r|}
\hline Logical & \multicolumn{4}{|c|}{ Shuffling } \\
Distribution & I & II & III & IV \\
\hline 1D & 8 & 12 & 10 & 9 \\
1S & 8 & 4 & 6 & 5 \\
$2 \mathrm{D}$ & 8 & 12 & 10 & 9 \\
$2 \mathrm{~S}$ & 8 & 4 & 6 & 5 \\
UN & 8 & 4 & 3 & 2 \\
\hline
\end{tabular}

We measure the average time taken by the four shuffling calls. Figure 12 shows the average execution times over three runs and the associated variability for the different logical distributions when searching the larger dataset on 256 nodes of Fusion. In the figure, we observe how in the first shuffling, 1D and $2 \mathrm{D}$ distributions take less time than the $1 \mathrm{~S}, 2 \mathrm{~S}$, and UN distributions do. In this shuffling phase, every compute node counts the octants at level 8. All the octkeys starting with the same 8 digits (e.g., 00000000, 00000001, 00000002) 950 are at the same level, and potentially there are $8^{8}$ many possible octants. In the $1 \mathrm{D}$ and $2 \mathrm{D}$ distributions, since the metadata populates only a small region of the space, there are fewer octants to count, so the scalar property aggregates that need to be communicated are smaller. In the 1S, $2 \mathrm{~S}$, and UN distributions, the metadata populates a larger number of tree nodes; thus, the scalar property aggregates that need to be communicated across nodes are larger. This phenomenon ultimately results in higher shuffling times.

The following shuffling phases (i.e., the $2 \mathrm{nd}$, 3rd, and 4th shuffling phases) explore different levels of the tree depending on whether a dense octant is found or not at level 8. Specifically, in their second shuffling, 1D and 2D explore level 12 of the tree (i.e., move down); this results in more octants to explore, larger messages to exchange, and longer shuffling times compared with the first 
shuffling phase at level 8. However, 1S, 2S, and UN explore level 4 of the tree (i.e., move up); this results in fewer octants to explore, smaller messages to exchange, and shorter shuffling times compared with the first shuffling.

In the third shuffling, 1D and 2D explore level 10 (i.e., move up); this shuffling deals with fewer octants compared with the second shuffling and, ultimately, shorter shuffling times than at level 12. This is not the case for $1 \mathrm{~S}$ and $2 \mathrm{~S}$ that explore level 6 (i.e., move down); this level has more octants and thus requires longer shuffling times compared with the second shuffling at level 4 . During the third shuffling, UN explores level 3; this is associated with fewer octants and shorter shuffle times than the second shuffling phase at level 4 .

In the fourth shuffling, 1D and 2D explore level 9 with its smaller number of octants compared to the previous shuffling and consequently requires shorter shuffle times. 1S, 2S explore level 5 with fewer octants compared with the third shuffling and shorter shuffling times. Finally, UN explores level 2; this level has fewer octants than the previous shuffle and also requires shorter shuffling times.

Empirically we observe how, at the large scale, communication times associated with shuffling calls are related to two factors in the search process: (1) the tree level to explore in the shuffling (i.e., the deeper the level, the more the number of octants, and the longer the shuffling times) and (2) the metadata distribution. For the latter factor, distributions where the metadata populates a smaller space (e.g., 1D and 2D) exhibit shuffling times that are smaller than distributions where metadata populates a larger space (e.g., 1S, 2S, and UN). Moreover, while at small scales the LocalToGlobal variation of our search does not show any sensitivity to the logical distributions, this is no longer true at the larger scales. Thus, in order to pursue the goal of insensitivity of performance to the distribution, new techniques and algorithms are needed.

\section{Accuracy}

The accuracy study aims to quantify the capability of our proposed mapping variations (i.e., the 3-D, 3-Dlog, and 6-D mappings) to capture and preserve lig- 
and conformation geometries. In other words, we want to assess whether our transformation of conformations into simple points preserves the knowledge on the conformations geometry and whether we can still acquire this knowledge on the most frequently sampled geometries despite their being generated across multiple nodes of a distributed-memory system. To this end we analyze the ligand conformations generated by real large-scale protein-ligand docking simulations that are part of the Docking@Home project.

\subsection{Platform}

The accuracy tests are executed in a small-scale cluster at the University of Delaware that is composed of 8 dual quad-core compute nodes (64 cores), each with two Intel Xeon $2.50 \mathrm{GHz}$ quad-core processors. A front-end node is connected to the compute nodes and is used for compilation and job submissions. A high-speed DDR InfiniBand interconnect is used for application and I/O traffic, and a Gigabit Ethernet interconnect for management traffic connects the compute and front-end nodes. The cluster mounts the real datasets generated by Docking@Home and thus provides easy access to the real data generated by the project at runtime.

\subsection{Datasets}

To test our proposed method for clustering ligand geometries, we ran docking trials on Docking@Home for 23 protein-ligand complexes for HIV protease (an aspartic acid protease protein), 21 protein-ligand complexes for trypsin (a serine protease protein), and 12 protein-ligand complexes for P38alpha kinase (a serine/threonine kinase protein) over five years. Figure 13 shows the three proteins. For each protein-ligand complex, we considered all the ligand conformations sampled with Docking@Home. The number of ligand conformations sampled for each complex is different, depending on the number of jobs successfully returned from the volunteer hosts and the number of conformations returned per job. On average, each complex contains around 210,000 ligand conformations. 
HIV protease (HIV PR) is a protein in the HIV virus that is essential for its replication in human cells. While building a new HIV virus inside the human cell, HIV PR cleaves some newly synthesized viral protein in a particular fashion. The cleaved pieces are required in order to build a mature HIV virus. HIV PR has been considered a therapeutic target to prevent HIV proliferation. A drug that can bind to the active site of HIV PR or deactivate it will eventually stop the replication of HIV virus in human cells. Such a drug is called a protease inhibitor. Several protease inhibitors (e.g., saquinavir, ritonavir, indinavir, and nelfinavir) are available for treating HIV infection [39.

Trypsin is a protease that breaks down other proteins in the digestive system. Recent studies suggest that inhibitors of trypsin can have potential application in breast cancer treatment. In this disease, trypsin-like proteases activate protease-activated receptor 2 (PAR2), a protein in the tumor cell membrane. While activated, PAR2 causes the degradation of the extracellular matrix, resulting in the spread of the tumor cell from one place to the other (metastasis). Drugs can act as inhibitors by deactivating the trypsin-like protease and are, therefore, potential agents capable of stopping the spread of breast cancer [40].

P38alpha is the most flexible protein among the three proteins considered. P38alpha is also known as SAPK2a and MAPK14. It is involved in the regulation of cellular stress responses as well as the control of proliferation and survival of many cell types. Several promising compounds that inhibit P38alpha are being investigated as potential therapies for arthritic and inflammatory diseases [41].

\subsection{Comparison across mappings and clustering}

To evaluate the capability of our method in capturing near-native conformations, we compare the accuracy of our clustering method based on the mapping of conformations into metadata and the N-D search with the accuracy of our previous work based on probabilistic hierarchical clustering [4] and the accuracy of the naïve selection approach based on only the lowest conformation energy also shown in [4. For each protein, its accuracy is the number of complexes with 
captured near-native conformations over the total number of complexes for that protein. Note that a near-native conformation has an RMSD from the experimentally observed conformation that is smaller than or equal to two Angstroms $(\AA)$.

For our clustering, we apply the three mapping methods described in Section 4 (i.e., 3-D, 3-Dlog, and 6-D mappings) to the real Docking@Home data and reduce the ligand geometries into metadata points. We compare and contrast the three mappings as they allow us to progressively capture a richer range of aspects related to the ligand geometry and location in the protein pocket. We consider two criteria when selecting the density threshold (i.e., the minimum number of metadata points in the tree nodes selected by the N-D search). The first criterion is to set the density threshold to a fixed number equal to 500 metadata points. In other words, we always select the densest tree node with at least 500 ligand conformations (i.e., octant in the case of the 3-D and 3-Dlog mappings or 6-D subspace in the case of the 6-D mapping). The second criterion is to set the density threshold equal to $0.5 \%$ of the number of ligands conformations in each protein-ligand complex dataset. For example, when the dataset contains 100,000 ligand conformations, the density threshold for this dataset is 500 points. When the dataset contains 500,000 ligand conformations, the density threshold for this dataset is 2,500 points. In both cases, we capture a near-native conformation if the arithmetic median of the conformations associated with the metadata point in the selected tree node is below or equal to $2 \AA$. The use of the median is preferred as the accuracy metric over the mean because it is less affected by extreme values, although the majority of our overall results are not very sensitive to whether the median or mean is used for selection.

For the probabilistic hierarchical clustering, the distance metric used to cluster each ligand is the RMSD of its atom coordinates versus all the other ligands already in the cluster. If a simulation converges, then the largest cluster with lower internal variance is likely the cluster that contains more near-native conformations. We capture a near-native conformation if the centroid of the selected cluster is a near-native conformation 4]. For the energy-based approach, we 
consider $100 \mathrm{D} @ \mathrm{H}$ conformations selected based on their lowest energy versus the same crystal structure, which we denote as the naïve approach. Here, we identify the near-native conformation if the arithmetic median of the lowest energy conformations is below or equal to $2 \AA$.

When using our method, or the probabilistic hierarchical clustering, or the energy-based approach, we perform the clustering and selection of the nearnative candidates without using any information on the crystal structures available for the complexes. The crystal structures play an important role only in the validation phase when, for each complex, we calculate the RMSD of the clustering candidate with respect to its crystal structure. Table 3 summarizes the accuracy of our method (N-D clustering) with a fixed density threshold equal to 500 and with density threshold equal to $0.5 \%$ of the dataset, for the probabilistic hierarchical clustering, and for the energy-based approach.

Our tests focus on three key aspects: (1) we study the accuracy of our methods versus more traditional but not scalable methods (e.g., the probabilistic hierarchical clustering) or traditional and scalable methods (e.g., the energybased approach); (2) we compare the impact of the different mappings on the accuracy when using our method; and (3) we quantify the impact of the different density thresholds on our N-D search and selection.

When comparing our methods with more traditional methods, we observe how, for all three proteins (i.e., HIV protease, trypsin, and P38alpha), our N-D clustering always gives better accuracy. In particular, for the HIV protease, our clustering (using 3-D, 3-Dlog, or 6-D mappings and the two density threshold selection criteria) captures all the 23 near-native conformations (100\%); the probabilistic hierarchical clustering method captures 20 of the 23 near-native conformations (87.0\%); and the naïve approach is able to identify only 8 of the 23 near-native conformations (34.8\%). For trypsin, our clustering captures 17 of the 21 near-native conformations (81.0\%); and the naïve approach identifies only 5 of the 21 near-native conformations (23.8\%). For the P38alpha kinase, the clustering captures 10 of the 12 near-native conformations $(83.3 \%)$; the probabilistic hierarchical approach captures 6 of the 12 near-native conformations 
(50.0\%); and the naïve approach identifies 1 of the 12 near-native conformations (0.8\%).

When mapping the ligand conformation into metadata using the three differ-

very low degrees of freedom). In this case, the 3-Dlog mapping method achieves better accuracy than the 3-D and 6-D methods achieve. In particular, for the 
trypsin protease, the 3-Dlog mapping method captures 16 of 21 (76.2\%), and 17 of $21(81.0 \%)$ near-native conformations using the two density threshold selection criteria. The reduced flexibility of the conformations explains why the 3-Dlog mapping works well for the associated clustering with trypsin but not for the other two proteins. Specifically, when a small and rigid ligand conformation is in a near-vertical position in a pocket, its slope is large. If the conformation position slightly changes, the slope also changes significantly, because of the projections. In the case of trypin, some conformations may have similar shapes and be in near-vertical positions, and their slopes may differ to the extent such that the mapping may not result in a dense enough subspace containing the metadata. By taking the log of the slopes, we slow the slope differences when dealing with vertical ligand conformations.

When dealing with different selection criteria (i.e., 500 points or a variable density threshold equal to $0.5 \%$ ), we observe how using a variable density threshold achieves better accuracy than using a fixed threshold equal to 500 for each individual dataset. Moreover, when a fixed density threshold equal to 500 is used, our method always outperforms the naïve approach for all the complexes; and our method also outperforms (for HIV and P38alpha) or is as accurate as (for trypsin) the probabilistic hierarchical clustering. Additionally, when a variable density threshold equal to $0.5 \%$ of the number of ligand conformations in the protein-ligand complex dataset is used, our N-D clustering always outperforms both the naïve approach and the probabilistic hierarchical clustering. In particular, for the HIV protease, when using a variable density, the 3-D, 3-Dlog, and 6-D achieve 100\%, 87.0\%, and 100\% accuracy, respectively; when using a fixed threshold, the 3-D, 3-Dlog, and 6-D achieve $91.3 \%, 73.9 \%$, and $100 \%$ accuracy, respectively. Similar results are observed for trypsin and P38alpha protease.

The 3-Dlog mapping method captures 16 of 21 (76.2\%), and 17 of $21(81.0 \%)$ near-native conformations using the two density threshold selection criteria. To be more specific, for the HIV protease, the N-D clustering captures all the near-native conformations $(100.0 \%), 20$ of $23(87.0 \%)$, and 23 of $23(100.0 \%)$ 
using the 3-D, 3-Dlog, and 6-D mappings, respectively. For trypsin, the N-D

1175 clustering captures 15 of the 21 near-native conformations (71.4\%), 17 of 21 (81.0\%), and 14 of 21 (66.7\%) using 3-D, 3-Dlog, and 6-D, respectively. For the P38alpha kinase, our N-D clustering captures 10 of the 12 near-native conformations (83.3\%), 8 of 12 (66.7\%), and 10 of 12 (83.3\%) using 3-D, 3-Dlog, and 6 -D, respectively. Overall, we observe how a variable density threshold better 1180 captures the convergence of docking simulations to a single ligand conformation that also matches crystal structure findings. 


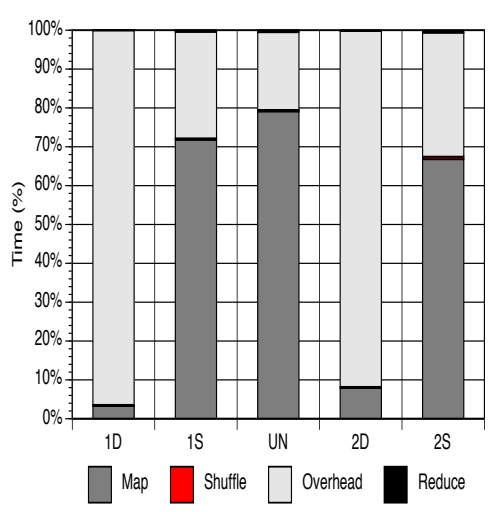

(a) Uniform, GlobalToLocal

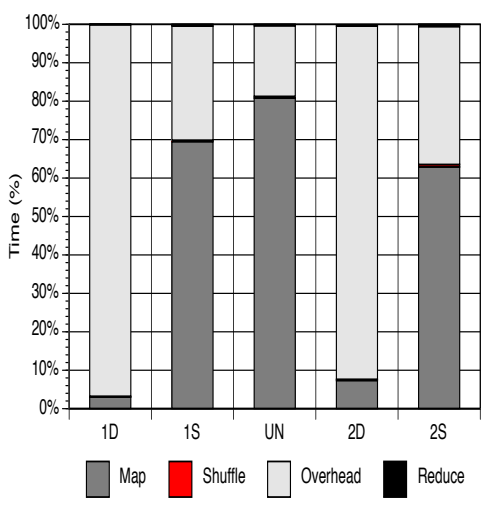

(c) Round-robin, GlobalToLocal

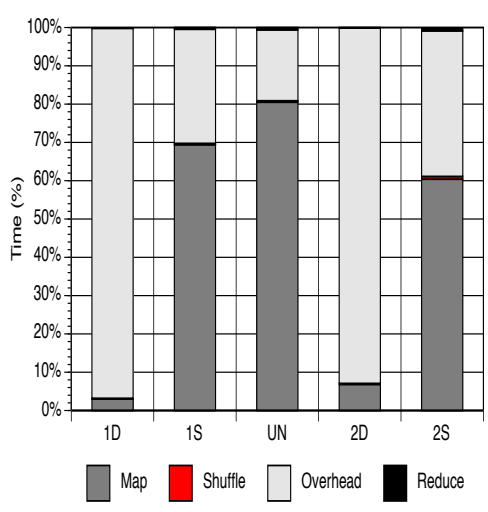

(e) Random, GlobalToLocal

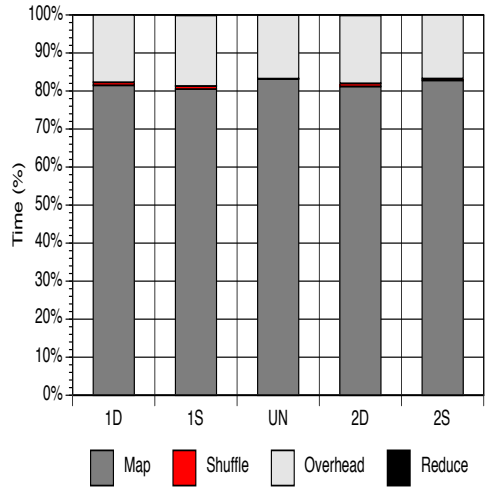

(b) Uniform, LocalToGlobal

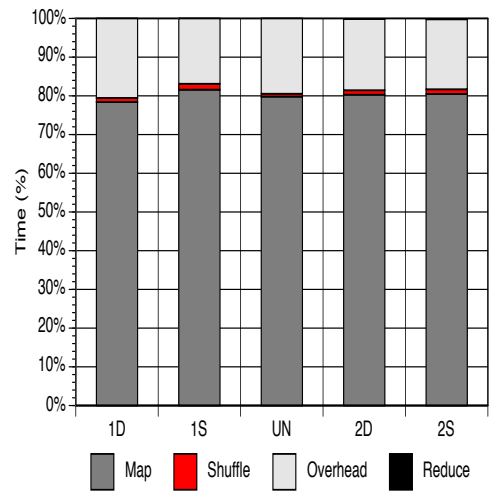

(d) Round-robin, LocalToGlobal

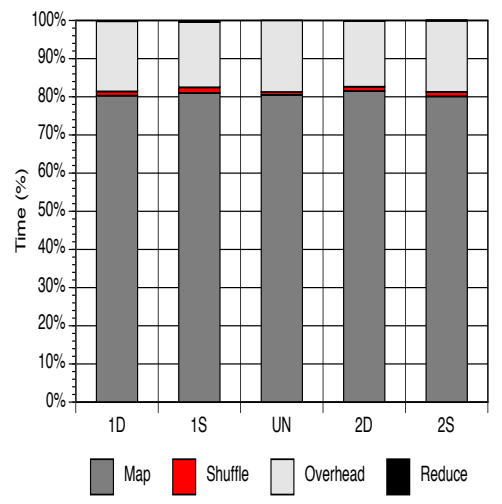

(f) Random, LocalToGlobal

Figure 9: Percentage of time for GlobalToLocal and LocalToGlobal for 5 logical distributions and the round-robin physical distribution. Similar results were observed for uniform and random distributions. 


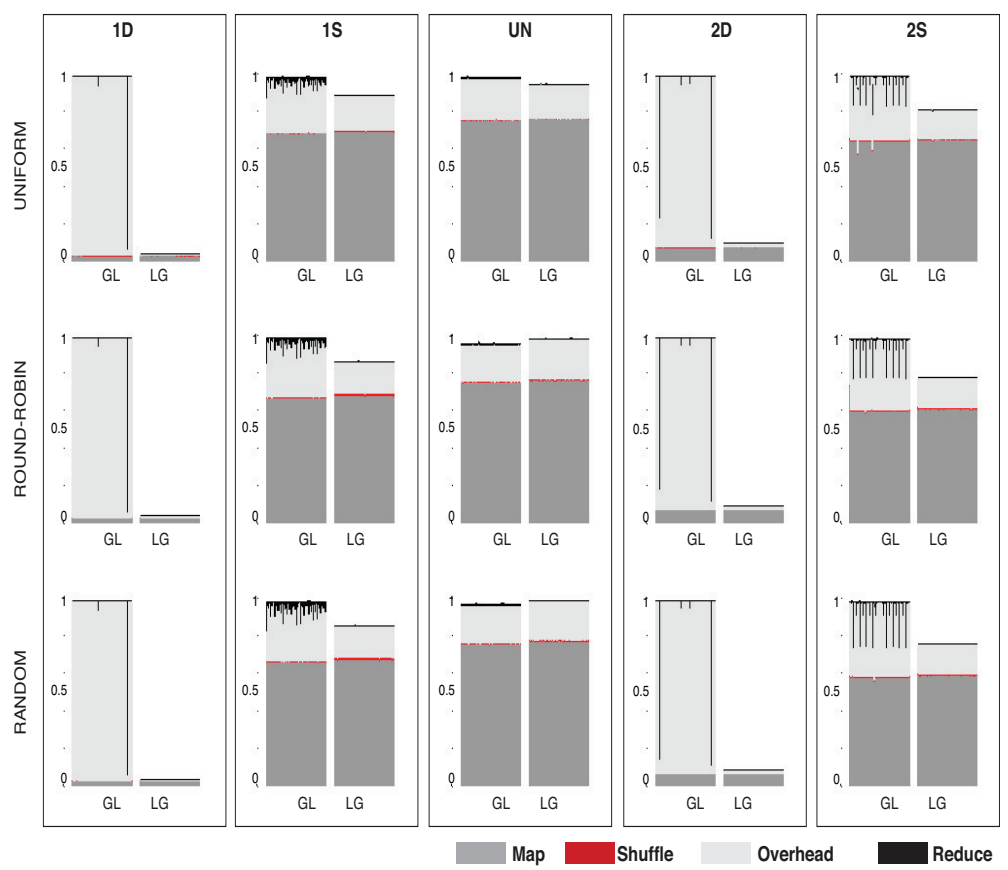

Figure 10: Per process times normalized w.r.t. the longest process per experiment for the round-robin and random physical distributions.

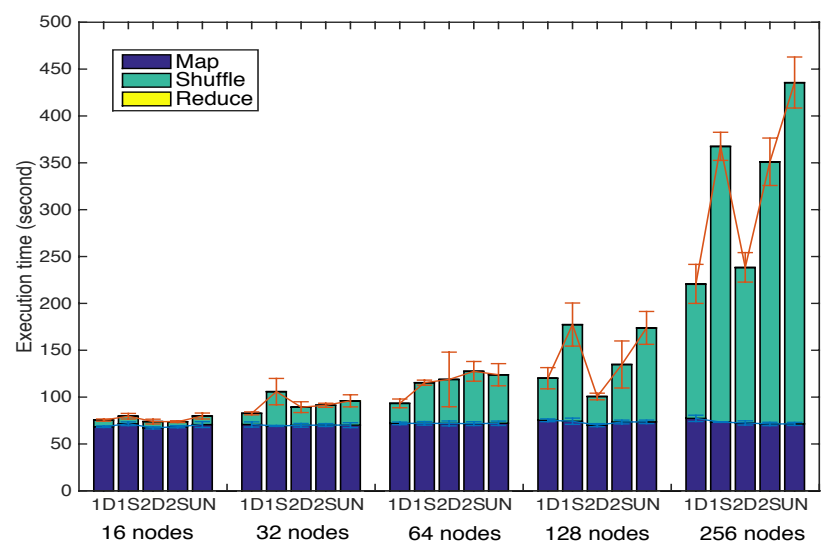

Figure 11: Averaged execution times in seconds and their variations for cut down in map, shuffling, and reduce times on Fusion with number of nodes ranging from 16 to 256 . 


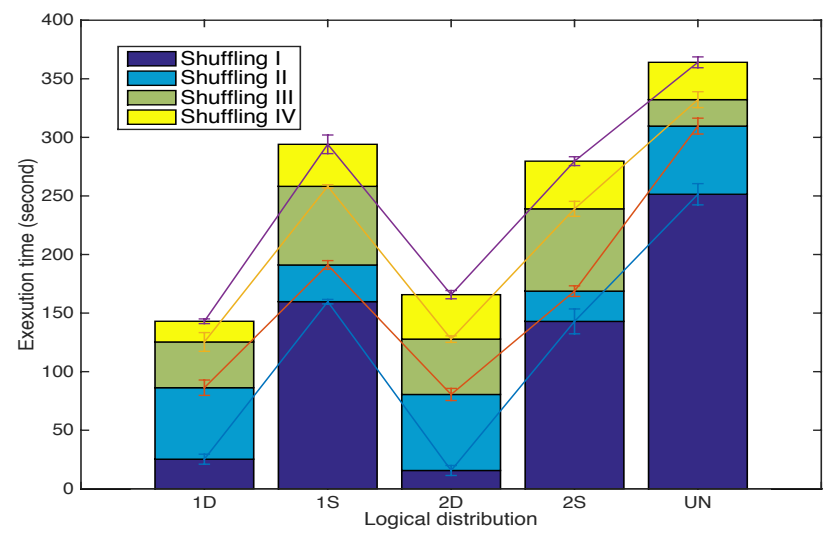

Figure 12: Averaged shuffling times and their variations for the four shuffling calls and the five distributions on 256 nodes of Fusion.

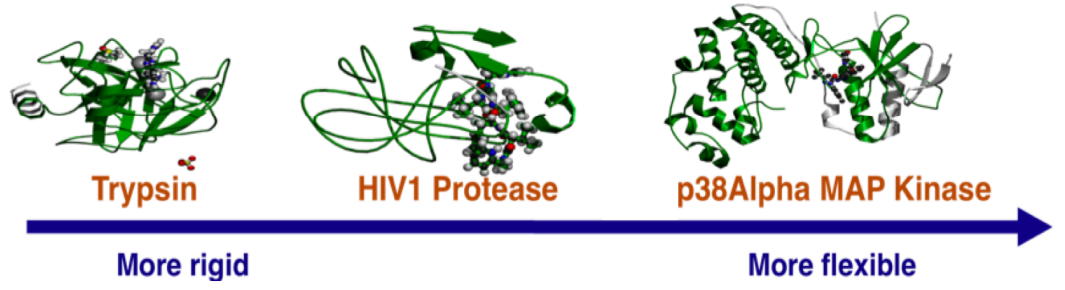

Figure 13: Three proteins whose results from the Docking@Home datasets are analyzed for this accuracy study. 
Table 3: Comparison of the number of hits for different scoring approaches: our clustering with density threshold equal to 500 and equal to $0.5 \%$ of the total number of points; a probabilistic hierarchical clustering; and an energy-based scoring method.

\begin{tabular}{|c|c|c|c|c|c|c|}
\hline \multirow[t]{2}{*}{ Protein } & \multicolumn{3}{|c|}{ N-D clustering with 500 points } & \multicolumn{3}{|c|}{ N-D clustering with $0.5 \%$ points } \\
\hline & $3-\mathrm{D}$ & 3-Dlog & $6-\mathrm{D}$ & $3-\mathrm{D}$ & 3-Dlog & $6-\mathrm{D}$ \\
\hline HIV & $21 / 23(91.3 \%)$ & $17 / 23(73.9 \%)$ & $23 / 23(100.0 \%)$ & $23 / 23(100.0 \%)$ & $20 / 23(87.0 \%)$ & $23 / 23(100.0 \%)$ \\
\hline Trypsin & $12 / 21(57.1 \%)$ & $16 / 21(76.2 \%)$ & $13 / 21(61.9 \%)$ & $15 / 21(71.4 \%)$ & $17 / 21(81.0 \%)$ & $14 / 21(66.7 \%)$ \\
\hline P38alpha & $9 / 12(75.0 \%)$ & $7 / 12(58.3 \%)$ & $10 / 12(83.3 \%)$ & $10 / 12(83.3 \%)$ & $8 / 12(66.7 \%)$ & $10 / 12(83.3 \%)$ \\
\hline All & $42 / 56(75.0 \%)$ & $40 / 56(71.4 \%)$ & $46 / 56(82.1 \%)$ & $48 / 56(85.7 \%)$ & $45 / 56(80.4 \%)$ & $47 / 56(83.9 \%)$ \\
\hline Protein & Hierarchical & Min. Energy & & & & \\
\hline \multicolumn{7}{|c|}{ cr } \\
\hline HIV & $20 / 23(87.0 \%)$ & $8 / 23(34.8 \%)$ & & & & \\
\hline Trypsin & $16 / 21(76.2 \%)$ & $5 / 21(23.8 \%)$ & & & & \\
\hline P38alpha & $6 / 12(50.0 \%)$ & $1 / 12(0.8 \%)$ & & & & \\
\hline All & $42 / 56(75.0 \%)$ & $14 / 56(25.0 \%)$ & & & & \\
\hline
\end{tabular}




\section{Conclusion and future work}

This paper focuses on avoiding data movement when analyzing scientific datasets comprising many complex data records distributed across nodes of 1185 a large distributed-memory system. We present a general method for data analysis - namely, clustering or classification - and apply it to structural biology datasets. The method performs a local, single preprocessing of the data to extract relevant properties (i.e., the geometrical properties including the shape and position) of a ligand conformation in a large dataset of millions of confirmations. We discuss variations of the general method including three ways to perform data reduction of the ligand conformations to metadata and two ways to perform the metadata space search.

We evaluate the performance of our method on 64 nodes of Gordon at the San Diego Supercomputer Center and 256 nodes of Fusion at Argonne National Laboratory, considering fifteen scenarios by combining five clustering configurations and three physical distributions of the data. At small scale on 64 nodes of Gordon, out method shows that when exchanging smaller messages of scalar property aggregates in the LocalToGlobal variation, the execution time is not sensitive to metadata distributions in the datasets: it varies only $3.4 \%$ across the five logical distributions. The GlobalToLocal variation, on the other hand, is sensitive to the metadata distributions. At large scale on 256 nodes of Fusion, our method shows that the data-shuffling stage dominates the execution time, and the shuffling time is affected by the metadata distribution as well as the level of trees explored in the N-dimensional tree. Moreover, we evaluate the accuracy of our method using three mapping methods and two density threshold selection criteria. The results on 56 ligands of three proteins show that our method can achieve 100\%, 81.0\%, and $85.7 \%$ clustering accuracy on protein HIV, trypsin, and P38alpha, respectively, whereas the traditional hierarchicalbased clustering achieves only $87.0 \%, 76.2 \%$, and $50.0 \%$ accuracy, respectively. Our accuracy analysis also reveals important insights into the fact that the 3-D and 6-D mapping methods work better on larger flexible ligands, whereas the 
3-Dlog mapping method works better on smaller, more rigid ligands.

Our variations are applicable to diverse scientific datasets and other distributed frameworks beyond the one considered in this paper and can result in scientific analyses that are not sensitive to data content and distribution. In the future, we plan to investigate and improve the scalability of our method at large scale by improving the performance of the shuffling stage of the MapReduceMPI library. Moreover, we plan to extend our metadata mapping method by using the angles of the three linear regression lines in the 2-D space that are in ${ }_{1220}$ a fixed range of 0 and $2 \pi$ and to apply our method in virtual screening.

\section{Acknowledgments}

This work was supported in part by NSF grant CCF1318445 and by the U.S. Department of Energy, Office of Science, under contract DE-AC02-06CH11357. This research used the Argonne Laboratory Computing Research Center "Fusion" cluster.

We thank Dr. Roger S. Armen (Thomas Jefferson University) and the Docking@Home volunteers for providing us with essential resources for our proteinligand docking simulations. The code of our clustering and sample ligands input data are available on github: https://github.com/TauferLab.

\section{References}

[1] M. Taufer, R. S. Armen, J. Chen, P. J. Teller, C. L. Brooks III, Computational multi-scale modeling in protein-ligand docking, IEEE Engineering in Medicine and Biology Magazine 28 (2) (2009) 58-69.

[2] W. Humphrey, A. Dalke, K. Schulten, VMD - Visual Molecular Dynamics, Journal of Molecular Graphics 14 (1996) 33-38.

[3] S. Ceri, R. Manthey, Chimera: A Model and Language for Active DOOD Systems, in: Proceedings of the 2nd East-West Database Workshop, Workshops in Computing, Springer, 1994, pp. 3-16. 
[4] T. Estrada, R. S. Armen, M. Taufer, Automatic Selection of Near-native Protein-ligand Conformations Using a Hierarchical Clustering and Volunteer Computing, in: Proceedings of the First ACM International Conference on Bioinformatics and Computational Biology, 2010, pp. 204-213.

[5] T. Estrada, B. Zhang, P. Cicotti, R. S. Armen, M. Taufer, Accurate Analysis of Large Datasets of Protein-ligand Binding Geometries Using Advanced Clustering Methods, Computers in Biology and Medicine 42 (7).

[6] B. Zhang, T. Estarda, P. Cicotti, M. Taufer, On Efficiently Capturing Scientific Properties in Distributed Big Data without Moving the Data - a Case Study in Distributed Structural Biology using MapReduce, in: Proceedings of the 2013 IEEE 16th International Conference on Computational Science and Engineering, 2013, pp. 817-822.

[7] B. Zhang, T. Estrada, P. Cicotti, P. Balaji, M. Taufer, Accurate Scoring of Drug Conformations at the Extreme Scale, in: Proceedings of the IEEE International Scalable Computing Challenges (SCALE 2015) - co-located with the 15th IEEE/ACM International Symposium on Cluster, Cloud and Grid Computing, 2015, pp. 817-822.

[8] O. Roche, R. Kiyama, C. L. B. III, Ligand-protein Database: Linking Protein-ligand Complex Structures to Binding Data, Journal of Medicinal Chemistry 44 (22) (2001) 3592-3598.

[9] R. Armen, E. May, M. Taufer, Protein Docking, Springer, 2011.

[10] A. Jain, Bias, Reporting, and Sharing: Computational Evaluations of Docking Methods, Journal of Computer-Aided Molecular Design 22 (3-4) (2008) 201-212.

[11] T. Estrada, B. Zhang, P. Cicotti, R. S. Armen, M. Taufer, Reengineering High-throughput Molecular Datasets for Scalable Clustering Using MapReduce, in: Proceedings of the 14th IEEE International Conference on High Performance Computing and Communications, 2012, pp. 351-359. 
[12] M. Taufer, R. S. Armen, J. Chen, P. J. Teller, C. L. Brooks, Computational Multiscale Modeling in Protein-ligand Docking, IEEE Engineering in Medicine and Biology Magazine 28 (2) (2009) 58-69.

[13] M. Rarey, B. Kramer, T. Lengauer, G. A. Klebe, A Fast Flexible Docking Method Using an Incremental Construction Algorithm, Journal of Molecular Biology 261 (3) (1996) 470-489.

[14] R. Abagyan, M. Totrov, D. Kuznetsov, A new Method for Protein Modeling and Design: Applications to Docking and Structure Prediction from the Distorted Native Conformation, Journal of Computational Chemistry 15 (5) (1994) 488-506.

[15] G. M. Morris, D. S. Goodsell, R. S. Halliday, R. Huey, W. E. Hart, R. K. Belew, A. J. Olson, Automated Docking Using a Lamarkian Genetic Algorithm and Empirical Binding Free Energy Function, Journal of Computational Chemistry 19 (14) (1998) 1639-1662.

[16] B. D. Bursulaya, M. Totrov, R. Abagyan, C. L. Brooks III, Comparative Study of Several Algorithms for Flexible Ligand Docking, Journal Of Computer-Aided Molecular Design 17 (11) (2003) 755-763.

[17] E. Perola, W. P. Walters, P. S. Charifson, A Detailed Comparison of Current Docking and Scoring Methods on Systems of Pharmaceutical Relevance, Proteins 56 (2) (2004) 235-249.

[18] P. Ferrara, H. Gohlke, D. Price, G. Klebe, C. L. Brooks III, Assessing Scoring Functions for Protein-ligand Interactions, Journal of Medicinal Chemistry 47 (12) (2004) 3032-3047.

[19] D. P. Anderson, BOINC: a System for Public-resource Computing and Storage, in: Proceedings of the 5th IEEE/ACM International Workshop on Grid Computing, 2004, pp. 4-10.

[20] M. S. Lee, M. Feig, F. R. Salsbury Jr., C. L. Brooks III, New Analytic Approximation to the Standard Molecular Volume Definition and its Appli- 
cation to Generalized Born Calculations, Journal of Computational Chemistry 24 (2003) 1348-1356.

[21] B. R. Brooks, R. E. Bruccoleri, B. D. Olafson, D. J. States, S. Swaminathan, M. Karplus, CHARMM: a Program for Macromolecular Energy Minimization, and Dynamics Calculations, Journal of Computational Chemistry 4 (2) (1983) 187-217.

[22] M. Taufer, M. Crowley, D. Price, A. A. Chien, C. L. Brooks III, Study of an Accurate and Fast Protein-ligand Docking Algorithm Based on Molecular Dynamics, Concurrency and Computation: Practice and Experience 17 (14) (2005) 1627-1641.

[23] S. J. Plimpton, K. D. Devine, MapReduce in MPI for Large-scale Graph Algorithms, Parallel Computing 37 (9).

[24] M. Zaharia, M. Chowdhury, M. J. Franklin, S. Shenker, I. Stoica, Spark: Cluster Computing with Working Sets, in: Proceedings of the 2nd USENIX Conference on Hot Topics in Cloud Computing, 2010, pp. 10-10.

[25] Apache Flink, https://flink.apache.org/index.html.

[26] S. Lorenzen, Y. Zhang, Identification of Near-native Structures by Clustering Protein Docking Conformations, PROTEINS: Structure, Function, and Bioinformatics 68 (2007) 187-194.

[27] G. Bouvier, N. Evrard-Todeschi, J. P. Girault, G. Bertho, Automatic Clustering of Docking Poses in Virtual Screening Process Using Self-organising Map, Bioinformatics Advance Access 26 (1) (2009) 53-60.

[28] M. W. Chang, R. K. Belew, K. S. Carroll, A. J. Olson, D. S. Goodsell, Empirical Entropic Contributions in Computational Docking: Evaluation in APS Reductase Complexes, Journal of Computational Chemistry 29 (11) (2008) 1753-1761. 
[29] H. G. Li, G. Q. Wu, X. G. Hu, J. Zhang, L. Li, X. Wu, K-means Clustering with Bagging and MapReduce, in: Proceedings of the 44th Hawaii International Conference on System Sciences, 2011, pp. 1-8.

[30] A. Ene, S. Im, B. Moseley, Fast Clustering Using MapReduce, in: Proceedings of the 17th ACM SIGKDD International Conference on Knowledge Discovery and Data Mining, 2011, pp. 681-689.

[31] R. L. F. Cordeiro, C. T. Jr, A. J. M. Traina, J. López, U. Kang, C. Faloutsos, Clustering Very Large Multi-dimensional Datasets with MapReduce, in: Proceedings of the 17th ACM SIGKDD International Conference on Knowledge Discovery and Data Mining, 2011, pp. 690-698.

[32] M. Hefeeda, F. Gao, W. Abd-Almageed, Distributed Approximate Spectral Clustering for Large-scale Datasets, in: Proceedings of the 21st International Symposium on High-Performance Parallel and Distributed Computing, 2012, pp. 223-234.

[33] Z. Rasheed, H. Rangwala, A Map-Reduce Framework for Clustering Metagenomes, in: Proceedings of the 13th IEEE International Workshop on High Performance Computational Biology, 2013, pp. 549-558.

[34] I. Gargantini, An Effective Way to Represent Quadtrees, Communications of the ACM 25 (12) (1982) 905-910.

[35] B. Moon, H. V. Jagadish, C. Faloutsos, J. H. Saltz, Analysis of the Clustering Properties of the Hilbert Space-filling Curve, IEEE Transactions on Knowledge and Data Engineering 13 (1) (2001) 124-141.

[36] T. Peterka, D. Goodell, R. Ross, H.-W. Shen, R. Thakur, A Configurable Algorithm for Parallel Image-compositing Applications, in: Proceedings of the Conference on High Performance Computing Networking, Storage and Analysis, 2009, pp. 4:1-4:10. 
[37] A. M. Caulfield, L. M. Grupp, S. Swanson, Gordon: Using Flash Memory to Build Fast, Power-efficient Clusters for Data-intensive Applications, ACM SIGARCH Computer Architecture News 37 (1) (2009) 217-228.

[41] Z. Wang, B. J. Canagarajah, J. C. Boehm, S. Kassisa, M. H. Cobb, P. Young, S. Abdel-Meguid, J. L. Adams, E. J. Goldsmith, Structural Basis of Inhibitor Selectivity in MAP Kinases, Structure 6 (1998) 1117-1128. 